\title{
Complexité des boréliens à coupes dénombrables
}

\author{
par
}

\author{
Dominique L e comte (Paris)
}

Résumé. Nous donnons, pour chaque niveau de complexité $\Gamma$, une caractérisation du type "test d'Hurewicz" des boréliens d'un produit de deux espaces polonais ayant toutes leurs coupes dénombrables ne pouvant pas être rendus $\Gamma$ par changement des deux topologies polonaises.

1. Introduction. Cet article fait suite à une étude entamée dans [Le1][Le3]. Il a pour objet de répondre à une conjecture faite dans ce dernier, et peut pour l'essentiel être lu indépendamment de ces articles. Cependant, la lecture préalable de ces articles peut éclairer plusieurs points techniques présents dans les arguments développés ici. Ces travaux se situent dans le cadre de la théorie descriptive des ensembles. Je renvoie le lecteur à $[\mathrm{Ku}]$ pour les notions de base de théorie descriptive classique et à $[\mathrm{Mo}]$ pour les notions de théorie descriptive effective. Pour déterminer la complexité exacte d'un borélien, on est amené à montrer qu'il n'est pas d'une classe de Baire donnée. Le théorème d'Hurewicz, rappelé ci-dessous, donne un critère pour la classe des $G_{\delta}$ (cf. [SR]) :

ThÉorème. Soient $X$ un espace polonais et $A$ un borélien de $X$. Les conditions suivantes sont équivalentes:

(a) Le borélien $A$ n'est pas $\Pi_{2}^{0}$.

(b) Il existe une injection continue $u: \mathbf{2}^{\omega} \rightarrow X$ telle que

$$
u^{-1}(A)=\left\{\alpha \in \mathbf{2}^{\omega} \mid \exists n \forall m \geq n \quad \alpha(m)=0\right\} .
$$

Cet exemple des suites nulles à partir d'un certain rang peut être remplacé par n'importe quel ensemble infini dénombrable sans point isolé de $\mathbf{2}^{\omega}$. Il est appelé "test d'Hurewicz" pour la classe des $G_{\delta}$. Ce théorème a été généralisé aux autres classes de Baire par A. Louveau et J. Saint Raymond (cf. [Lo-SR]).

2000 Mathematics Subject Classification: 54H05, 03E15, 26A21, 28A05. 
On s'intéresse ici à une hiérarchie analogue à celle de Baire, sauf qu'au lieu de partir des ouverts-fermés d'un espace polonais de dimension 0 , on part des produits de deux boréliens, chacun d'entre eux étant inclus dans un espace polonais. L'analogie devient plus claire quand on sait qu'étant donnés un espace polonais $X$ et un borélien $A$ de $X$, on peut trouver une topologie polonaise plus fine que la topologie initiale sur $X$ (topologie ayant donc les mêmes boréliens), de dimension 0 , et qui rende $A$ ouvert-fermé. Pour notre problème, le fait de travailler dans les espaces de dimension 0 n'est donc pas une restriction réelle. La définition qui suit apparaît alors naturelle.

DÉfinition. Soient $X$ et $Y$ des espaces polonais, et $A$ un borélien de $X \times Y$. Si $\Gamma$ est une classe de Baire, on dira que $A$ est potentiellement dans $\Gamma$ (ce qu'on notera $A \in \operatorname{pot}(\Gamma)$ ) s'il existe des topologies polonaises de dimension $0, \sigma(\operatorname{sur} X)$ et $\tau(\operatorname{sur} Y)$, plus fines que les topologies initiales, telles que $A$, considéré comme partie de $(X, \sigma) \times(Y, \tau)$, soit dans $\Gamma$.

La motivation pour l'étude de ces classes de Wadge potentielles trouve son origine dans l'étude des relations d'équivalence boréliennes, et plus précisément dans l'étude du pré-ordre suivant sur la collection des relations d'équivalence boréliennes définies sur un espace polonais :

$$
E \leq F \Leftrightarrow \exists f \text { borélienne } E=(f \times f)^{-1}(F) .
$$

A l'aide de la notion de classe de Wadge potentielle, A. Louveau montre dans [Lo3] que la collection des relations d'équivalence $\boldsymbol{\Sigma}_{\xi}^{0}$ n'est pas co-finale, et il en déduit qu'il n'existe pas de relation maximum pour $\leq$.

On cherche à établir des résultats analogues au théorème d'Hurewicz pour les classes de Baire potentielles. Le résultat principal de cet article établit l'analogue du théorème d'Hurewicz pour la classe des ensembles potentiellement $G_{\delta}$. Dans [Le3], il y a la

Conjecture. Il existe un borélien $B$ de $\mathbf{2}^{\omega} \times \mathbf{2}^{\omega}$ tel que pour tous espaces polonais $X$ et $Y$, et pour tout borélien $A$ de $X \times Y$ à coupes verticales dénombrables, on a l'équivalence entre les conditions suivantes:

(a) Le borélien $A$ n'est pas $\operatorname{pot}\left(\boldsymbol{\Pi}_{2}^{0}\right)$.

(b) Il existe des fonctions continues $u: \mathbf{2}^{\omega} \rightarrow X$ et $v: \mathbf{2}^{\omega} \rightarrow Y$ telles que $\bar{B} \cap(u \times v)^{-1}(A)=B$.

L'essentiel de cet article va consister à analyser les boréliens non $\operatorname{pot}\left(\boldsymbol{\Pi}_{2}^{0}\right)$ ayant leurs coupes horizontales et verticales dénombrables pour arriver progressivement à montrer le

THÉORÈME 7. Il existe un borélien $B$ de $\omega^{\omega} \times \omega^{\omega}$ tel que pour tous espaces polonais $X$ et $Y$, et pour tout borélien $A$ de $X \times Y$ dont les coupes horizontales et verticales sont dénombrables, on a l'équivalence entre les conditions suivantes: 
(a) Le borélien $A$ n'est pas $\operatorname{pot}\left(\mathbf{\Pi}_{2}^{0}\right)$.

(b) Il existe $u: \omega^{\omega} \rightarrow X$ et $v: \omega^{\omega} \rightarrow Y$, homéomorphismes sur leurs images, tels que $\bar{B} \cap(u \times v)^{-1}(A)=B$.

Ce borélien $B$ sera une "version uniforme" du test d'Hurewicz, c'est-àdire un ensemble dont toutes les coupes verticales sont infinies dénombrables sans point isolé. Plus précisément, $B$ sera réunion dénombrable de graphes d'homéomorphismes de domaine ouvert-fermé. On verra aussi qu'essentiellement, dans tout borélien ayant ses coupes horizontales et verticales dénombrables et n'étant pas $\operatorname{pot}\left(\boldsymbol{\Pi}_{2}^{0}\right)$, on peut trouver, à un changement de topologie près, une telle réunion se réduisant à $A$ au sens du théorème 7 . On cherchera entre autres à réduire de telles réunions entre elles.

L'hypothèse de dénombrabilité des coupes dans le théorème 7 peut sembler moins naturelle que par exemple l'hypothèse " $A$ est $\operatorname{pot}\left(\boldsymbol{\Sigma}_{2}^{0}\right)$ ". Mais cette dernière n'est pas suffisante. En effet, les boréliens à coupes verticales (ou horizontales) dénombrables sont $\operatorname{pot}\left(\boldsymbol{\Sigma}_{2}^{0}\right)$ (voir [Lo2]). Nous montrons que le théorème 7 devient faux si on suppose seulement $A$ à coupes verticales dénombrables, en utilisant l'injectivité de $u$ et $v$.

Pour terminer cette introduction, nous plaçons le théorème 7 dans un contexte plus général. Il vient en effet compléter l'étude des boréliens à coupes verticales dénombrables commencée dans [Le3]. Je renvoie le lecteur à cet article pour les rappels concernant la hiérarchie de Wadge, qui affine celle de Baire. On peut montrer que les seules classes de Wadge non stables par passage au complémentaire contenues dans $\boldsymbol{\Delta}_{2}^{0}=\boldsymbol{\Sigma}_{2}^{0} \cap \boldsymbol{\Pi}_{2}^{0}$ sont les différences transfinies d'ouverts. On peut définir sans problème la notion d'ensemble potentiellement dans $\Gamma$, où $\Gamma$ est une classe de Wadge, en utilisant la même définition que précédemment. L'analogue du théorème 7 pour les différences transfinies d'ouverts est montré dans [Le3] (voir théorèmes 3.5 et 3.6), à ceci près que l'hypothèse est moins forte (" $A$ est potentiellement $\boldsymbol{\Delta}_{3}^{0}$ " au lieu de " $A$ a ses coupes horizontales et verticales dénombrables"), et que la conclusion est moins forte (on n'a pas l'injectivité des fonctions de réduction). Comme conséquence de ces résultats, on obtient le résultat de synthèse suivant :

Corollaire 9. Soit $\Gamma$ une classe de Wadge non stable par passage au complémentaire. Alors il existe un borélien $B_{\Gamma}$ de $\omega^{\omega} \times \omega^{\omega}$ et un fermé $F_{\Gamma}$ contenant $B_{\Gamma}$, tels que pour tous espaces polonais $X$ et $Y$, et pour tout borélien $A$ de $X \times Y$ ayant ses coupes horizontales et verticales dénombrables, on a l'équivalence entre les conditions suivantes:

(a) Le borélien $A$ n'est pas pot $(\Gamma)$.

(b) Il existe des fonctions continues $u: \omega^{\omega} \rightarrow X$ et $v: \omega^{\omega} \rightarrow Y$ telles que $F_{\Gamma} \cap(u \times v)^{-1}(A)=B_{\Gamma}$. 
Il est à noter que $B_{\Gamma}$ et $F_{\Gamma}$ vont être donnés de manière explicite, et que $B_{\Gamma}$ a ses coupes horizontales et verticales dénombrables si $\Gamma \subseteq \mathbf{\Pi}_{2}^{0}$, ce qui est le cas significatif. Donc en particulier $B_{\Gamma} \notin \operatorname{pot}(\Gamma)$ si $\Gamma \subseteq \boldsymbol{\Pi}_{2}^{0}$. D'autre part, si $\Gamma$ est auto-duale (c'est-à-dire si $\Gamma$ est stable par passage au complémentaire), ne pas être dans $\Gamma$ signifie ne pas être dans l'une des deux classes non auto-duales succédant à $\Gamma$ dans l'ordre de Wadge (l'inclusion des classes). L'étude des classes de Wadge auto-duales peut donc être ramenée à celle des classes de Wadge non auto-duales.

QUESTION. Un problème ouvert est de savoir si on peut supprimer l'hypothèse " $A$ a ses coupes horizontales et verticales dénombrables" dans le corollaire 9 .

2. Analyse des boréliens à coupes dénombrables n'étant pas $\operatorname{pot}\left(\boldsymbol{\Pi}_{2}^{0}\right)$. La définition qui suit donne un sens précis à l'expression "version uniforme du test d'Hurewicz" évoquée dans l'introduction.

DÉfinition. On dira que $\left(Z, T,\left(g_{m, p}\right)_{(m, p) \in \omega^{2}}\right)$ est une situation générale si :

(a) $Z$ et $T$ sont des espaces polonais parfaits de dimension 0 non vides.

(b) $g_{m, p}$ est un homéomorphisme de domaine $D_{g_{m, p}}$ (respectivement d'image) ouvert-fermé de $Z$ (respectivement de $T$ ).

(c) Pour $m \in \omega,\left(D_{g_{m, p}}\right)_{p \in \omega}$ est une suite de domaines deux à deux disjoints dont la réunion est dense dans $Z$. On note $g_{m}$ la fonction obtenue par recollement des $g_{m, p}$, pour $p$ entier.

(d) Il existe un $G_{\delta}$ dense $G(g)$ de $\bigcap_{m \in \omega} D_{g_{m}}$ tel que l'ensemble $g[x]:=\left\{g_{m}(x) \mid m \in \omega\right\}$ soit sans point isolé, pour tout $x$ de $G(g)$.

L'idée va être de chercher le borélien $B$ du théorème 7 sous la forme $\bigcup_{m \in \omega} \operatorname{Gr}\left(g_{m}\lceil G(g))\right.$, où $\left(Z, T,\left(g_{m, p}\right)_{(m, p) \in \omega^{2}}\right)$ est une situation générale. Et aussi de montrer que dans chaque borélien $A$ dont toutes les coupes sont dénombrables et n'étant pas pot $\left(\boldsymbol{\Pi}_{2}^{0}\right)$, on peut trouver, à un changement de topologie près, une telle réunion se réduisant à $A$ au sens du théorème 7 . On va donc être amené à réduire une situation générale à une autre. C'est l'objet du théorème 1 qui suit. Il se trouve que pour assurer l'existence d'une telle réduction, il nous faut des conditions supplémentaires, aussi bien au départ qu'à l'arrivée. D'où les deux définitions qui suivent. Toutes les conditions supplémentaires de ces définitions seront utilisées dans la preuve du théorème 1 qui suit, à l'exception de la condition (b) d'une situation d'arrivée, dont l'intérêt apparaîtra plus tard.

DÉfinition. On dira que $\left(Z, T,\left(g_{m, p}\right)_{(m, p) \in \omega^{2}}\right)$ est une situation d'arrivée si :

(a) $\left(Z, T,\left(g_{m, p}\right)_{(m, p) \in \omega^{2}}\right)$ est une situation générale. 
(b) Le diamètre du domaine et de l'image de $g_{m, p}$ vaut au plus $2^{-\Delta(m, p)}$, où $\Delta: \omega^{2} \rightarrow \omega$ est injective.

(c) Pour tout entier non nul $k$ et pour toute suite $u$ dans $\left(\omega^{2}\right)^{2 k}$, on a l'implication

$$
\begin{array}{r}
\left(\exists U \in \Delta_{1}^{0}, \emptyset \neq U \subseteq Z \forall x \in U \quad g_{u(0)}^{-1} g_{u(1)} \ldots g_{u(2 k-2)}^{-1} g_{u(2 k-1)}(x)=x\right) \\
\Rightarrow(\exists i<2 k-1 \quad u(i)=u(i+1)) .
\end{array}
$$

DÉfinition. On dira que $\left(F,\left(f_{n, p}\right)_{(n, p) \in \omega^{2}}\right)$ est une situation de départ si :

(a) $\left(F, F,\left(f_{n, p}\right)_{(n, p) \in \omega^{2}}\right)$ est une situation générale.

(b) $F=\prod_{i \in \omega} A_{i} \subseteq \omega^{\omega}$, où chaque $A_{i} \subseteq \omega$ est fini.

(c) Pour $x$ dans $D_{f_{n}}$, on a $x<_{\text {lex }} f_{n}(x)$, et pour $n, p$ entiers, il existe un entier $q(n, p)$ tel que si $q>q(n, p)$ et $x$ est dans $D_{f_{n, p}}, f_{n, p}(x)(q)=x(q)$.

(d) Pour $(x, y)$ dans $\left(\bigcap_{n \in \omega} D_{f_{n}} \times F\right) \cap \overline{\bigcup_{n \in \omega} \operatorname{Gr}\left(f_{n}\right)}$, il existe $\left(y_{k}\right)$ tendant vers $y$ telle que $\left(x, y_{k}\right)$ appartienne à $\bigcup_{n \in \omega} \operatorname{Gr}\left(f_{n}\right)$ pour tout entier $k$, et $x \neq y$.

(e) On définit des relations sur $\bigcup_{p \in \omega}\left(\prod_{i<p} A_{i}\right), n$ étant entier, par

$$
\begin{aligned}
s \mathfrak{R}_{n} t & \Leftrightarrow|s|=|t| \text { et } s \leq_{\text {lex }} t \text { et }\left(N_{s} \times N_{t}\right) \cap \operatorname{Gr}\left(f_{n}\right) \neq \emptyset, \\
s \mathfrak{R} t & \Leftrightarrow \exists n \in \omega s \mathfrak{R}_{n} t .
\end{aligned}
$$

Si $s \Re t$, on pose $\psi(s, t):=\min \left\{n \in \omega \mid s \Re_{n} t\right\}$. On demande :

(i) $[s \mathfrak{R} s \Rightarrow \psi(s, s)=0]$ et $[s \frown j \mathfrak{R} t \frown j \Rightarrow \psi(s \frown j, t \frown j)=\psi(s, t)]$.

On définit ensuite la relation symétrique engendrée par $\mathfrak{R}$ :

$$
s \mathfrak{T} t \Leftrightarrow s \mathfrak{R} t \text { ou } t \mathfrak{R} s .
$$

On dira que $u \in\left[\bigcup_{p \in \omega}\left(\prod_{i<p} A_{i}\right)\right]^{<\omega} \backslash\{\emptyset\}$ est une $\mathfrak{T}$-chaîne si pour tout $j<|u|-1, u(j) \mathfrak{T} u(j+1)$. On définit la relation d'équivalence engendrée par $\mathfrak{R}$ :

$$
s \mathfrak{E} t \Leftrightarrow \exists u \text { T-chaîne } u(0)=s \text { et } u(|u|-1)=t .
$$

On demande :

(ii) Si $s \mathfrak{E} t$, il existe une unique $\mathfrak{T}$-chaîne $u$, sans répétition de termes, telle que $u(0)=s$ et $u(|u|-1)=t$.

THÉORÈme 1. Soient $\left(F,\left(f_{n, p}\right)_{(n, p) \in \omega^{2}}\right)$ une situation de départ et $\left(Z, T,\left(g_{m, p}\right)_{(m, p) \in \omega^{2}}\right)$ une situation d'arrivée. Alors il existe des injections continues $u: F \rightarrow G(g)$ et $v: F \rightarrow T$ telles que :

(a) Pour tout $(x, y)$ dans $\bigcup_{n \in \omega} \operatorname{Gr}\left(f_{n}\right), v(y)$ est dans $g[u(x)]$.

(b) Pour tout $(x, y)$ dans $\left(\bigcap_{n \in \omega} D_{f_{n}} \times F\right) \cap \overline{\bigcup_{n \in \omega} \operatorname{Gr}\left(f_{n}\right)} \backslash \bigcup_{n \in \omega} \operatorname{Gr}\left(f_{n}\right)$, $v(y)$ est dans $\overline{g[u(x)]} \backslash g[u(x)]$. 
Démonstration. Le schéma de la preuve est semblable à celui de la preuve du théorème 2.12 de [Le3] ; la condition (iv) ci-dessous apporte des complications. On va construire :

- Une suite $\left(U_{s}\right)_{s \in \cup_{p \in \omega} \prod_{i<p} A_{i}}$ d'ouverts-fermés non vides de $Z$.

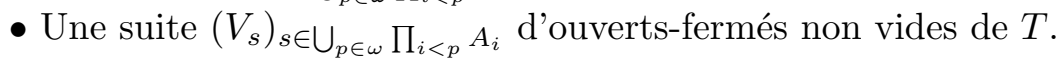

- Une injection $\Phi: \bigcup_{p \in \omega}\left(\prod_{i<p} A_{i}\right)^{2} \rightarrow \omega^{2}$.

On pose, si $s \mathfrak{R} t$,

$$
\begin{aligned}
n(s, t) & :=\min \{n \in \omega \cup\{-1\}|\forall n<i<| s \mid \quad s(i)=t(i)\}, \\
w(s, t) & :=\Phi(s\lceil(n(s, t)+1), t\lceil(n(s, t)+1)) .
\end{aligned}
$$

La première coordonnée de $w(s, t)$ sera notée $w_{0}(s, t)$. Soit $\left(O_{q}\right)$ une suite d'ouverts de $Z$ telle que $G(g):=\bigcap_{q \in \omega} O_{q}$. On demande à ces objets de vérifier les conditions suivantes:

(i) $U_{s \frown i} \times V_{s \frown i} \subseteq\left(U_{s} \cap \bigcap_{q \leq|s|} O_{q}\right) \times V_{s}$.

(ii) $\delta\left(U_{s}\right), \delta\left(V_{s}\right) \leq 2^{-|s|}$.

(iii) $s \mathfrak{R} t \Rightarrow V_{t}=g_{w(s, t)}\left[U_{s}\right]$ et $w_{0}(s, t) \geq \psi(s, t)$.

(iv) $\left(|s|=|t|, s<_{\text {lex }} t\right.$ et $r<|s|$ vérifie $\left.\forall q \leq r \quad s \quad \Re_{q} t\right)$ $\Rightarrow\left(\left(U_{s} \times V_{t}\right) \cap \bigcup_{q \leq r} \operatorname{Gr}\left(g_{q}\right)=\emptyset\right)$.

(v) $U_{s \frown i} \cap U_{s \frown j}=V_{s \frown i} \cap V_{s \frown j}=\emptyset$ si $i \neq j$.

Admettons ceci réalisé. On définit les injections continues $u$ et $v$ par les formules

$$
\{u(\alpha)\}:=\bigcap_{n \in \omega} U_{\alpha\lceil n}, \quad\{v(\alpha)\}:=\bigcap_{n \in \omega} V_{\alpha\lceil n} .
$$

Si $y=f_{n}(x)$, pour tout entier $m$ on a que $x\lceil m \mathfrak{R} y\lceil m$, et il existe un entier $m_{0}$ tel que si $m \geq m_{0}, n\left(x\left\lceil m, y\lceil m)=n\left(x\left\lceil m_{0}, y\left\lceil m_{0}\right)\right.\right.\right.\right.$, puisque $x(q)$ et $y(q)$ coïncident à partir d'un certain rang (on utilise la condition (c) d'une situation de départ). Posons $w_{0}:=w\left(x\left\lceil m_{0}, y\left\lceil m_{0}\right)\right.\right.$. Par (iii) on a $V_{y\lceil m}=g_{w_{0}}\left[U_{x\lceil m}\right]$ si $m \geq m_{0}$. D'où

$$
g_{w_{0}}(u(x)) \in g_{w_{0}}\left[\bigcap_{m \geq m_{0}} U_{x\lceil m}\right] \subseteq \bigcap_{m \geq m_{0}} g_{w_{0}}\left[U_{x\lceil m}\right]=\bigcap_{m \geq m_{0}} V_{y\lceil m}=\{v(y)\}
$$

et $(u(x), v(y)) \in \operatorname{Gr}\left(g_{w_{0}}\right)$.

Si maintenant $(x, y) \in\left(\bigcap_{n \in \omega} D_{f_{n}} \times F\right) \cap \overline{\bigcup_{n \in \omega} \operatorname{Gr}\left(f_{n}\right)} \backslash \bigcup_{n \in \omega} \operatorname{Gr}\left(f_{n}\right)$, soit $\left(y_{k}\right)$ telle que $\left(x, y_{k}\right) \in \bigcup_{n \in \omega} \operatorname{Gr}\left(f_{n}\right)$ et $y_{k}$ tende vers $y$ (on utilise la condition (d) d'une situation de départ). Par ce qui précède, on a que $\left(u(x), v\left(y_{k}\right)\right) \in \bigcup_{m \in \omega} \operatorname{Gr}\left(g_{m}\right)$, d'où $v\left(y_{k}\right) \in g[u(x)]$ et $v(y) \in \overline{g[u(x)]}$, par continuité de $v$. Pour tout entier $r$, il existe un entier $p(r)>r$ tel que pour $q \leq r, x\left\lceil p(r) \mathfrak{R}_{q} y\left\lceil p(r)\right.\right.$ et $x\left\lceil p(r)<_{\text {lex }} y\lceil p(r)\right.$ puisque $x \neq y$; par (iv) on a donc $\left(U_{x\lceil p(r)} \times V_{y\lceil p(r)}\right) \cap \bigcup_{q \leq r} \operatorname{Gr}\left(g_{q}\right)=\emptyset$ et $(u(x), v(y)) \notin \bigcup_{q \leq r} \operatorname{Gr}\left(g_{q}\right)$, donc $v(y) \notin g[u(x)]$. 
Montrons donc que la construction est possible. On pose $U_{\emptyset}:=D_{g_{0,0}}$, $V_{\emptyset}:=g_{0,0}\left[U_{\emptyset}\right]$, et $\Phi(\emptyset, \emptyset):=(0,0)$ (on peut supposer que $D_{g_{0,0}} \neq \emptyset$ ). Admettons avoir construit $U_{s}, V_{s}$ et $\Phi(s, t)$ pour $|s|,|t| \leq p$ vérifiant (i)-(v), et soient $s \in \prod_{j<p} A_{j}$ et $i \in A_{p}$. On note, si $\widetilde{s} \mathfrak{E} \widetilde{t}, \mathfrak{T}(\widetilde{s}, \widetilde{t})$ l'unique $\mathfrak{T}$-chaîne sans répétition de termes $c$ telle que $c(0)=\widetilde{s}$ et $c(|c|-1)=\widetilde{t}$, qui existe par la condition (e)(ii) d'une situation de départ.

La relation $\mathfrak{T}$ définit sur $\mathfrak{E}(s \frown i)$ une structure d'arbre, de sommet $s \frown i$. On va essentiellement construire les ouverts-fermés cherchés par récurrence sur les niveaux de cet arbre. Si $k$ est entier naturel, on pose $H_{k}:=\{z \in \mathfrak{E}(s \frown i)|| \mathfrak{T}(z, s \frown i) \mid=k+1\}$. Alors $H_{k}$ et le nombre de $H_{k}$ non vides sont finis, puisque les classes d'équivalence de $\mathfrak{E}$ sont finies. De plus, $H_{k}$ est non vide si $H_{k+1}$ l'est, donc on peut trouver $q$ tel que $H_{0}, \ldots, H_{q}$ soient non vides et $H_{k}$ soit vide si $k>q$. Posons

$$
H_{k}:=\left\{z_{(k, 1)}, \ldots, z_{\left(k, p_{k}\right)}\right\}, \phi:\left\{\begin{aligned}
\bigcup_{k \leq q}\{k\} \times\left\{1, \ldots, p_{k}\right\} & \rightarrow \omega \\
(k, r) & \mapsto\left(\sum_{i<k} p_{i}\right)+r .
\end{aligned}\right.
$$

On a donc

$\operatorname{Im}(\phi)=\left\{p_{0}=1, p_{0}+1, \ldots, p_{0}+p_{1}, \ldots, p_{0}+\ldots+p_{q-1}+1, \ldots, p_{0}+\ldots+p_{q}\right\}$.

On va construire par récurrence sur $n \in\left\{1, \ldots, p_{0}+\ldots+p_{q}\right\}$, et pour $k \in\{1, \ldots, n\}$, des ouverts-fermés non vides $U_{k}^{n}$ et $V_{k}^{n}$ tels que, si l'on pose $w(k, l):=w\left(z_{\phi^{-1}(k)}, z_{\phi^{-1}(l)}\right)$, on ait :

(1) $U_{k}^{n} \times V_{k}^{n} \subseteq\left(U_{z_{\phi^{-1}(k)}}\left\lceil p \cap \bigcap_{q \leq p} O_{q}\right) \times V_{z_{\phi^{-1}(k)}\lceil p}\right.$.

(2) $\delta\left(U_{k}^{n}\right), \delta\left(V_{k}^{n}\right) \leq 2^{-p-1}$.

(3) Si $k, l \in\{1, \ldots, n\}$, alors $V_{k}^{n}=g_{0,0}\left[U_{k}^{n}\right]$ et si de plus $z_{\phi^{-1}(k)} \mathfrak{R} z_{\phi^{-1}(l)}$, alors $V_{l}^{n}=g_{w(k, l)}\left[U_{k}^{n}\right]$ et $w_{0}(k, l) \geq \psi\left(z_{\phi^{-1}(k)}, z_{\phi^{-1}(l)}\right)$.

(4) Si $1 \leq k, l \leq n, z_{\phi^{-1}(k)}<_{\text {lex }} z_{\phi^{-1}(l)}$ et $r \leq p$ vérifie $z_{\phi^{-1}(k)} \mathfrak{R}_{q} z_{\phi^{-1}(l)}$ pour $q \leq r$, alors $\left(U_{k}^{n} \times V_{l}^{n}\right) \cap \bigcup_{q \leq r} \operatorname{Gr}\left(g_{q}\right)=\emptyset$.

(5) Si $1 \leq k, l \leq n$ et $k \neq l$, alors $U_{k}^{n} \cap U_{l}^{n}=V_{k}^{n} \cap V_{l}^{n}=\emptyset$.

(6) Si $1 \leq k \leq n$, alors $U_{k}^{n+1} \times V_{k}^{n+1} \subseteq U_{k}^{n} \times V_{k}^{n}$.

Admettons cette construction effectuée. On voudrait alors poser $U_{z_{\phi^{-1}(k)}}:=U_{k}^{p_{0}+\ldots+p_{q}}, V_{z_{\phi^{-1}(k)}}:=V_{k}^{p_{0}+\ldots+p_{q}}$ et si $\Phi\left(z, z^{\prime}\right)$ n'est pas encore défini, on le définirait en assurant l'injectivité de $\Phi$ et la seconde partie de la condition (iii); on obtiendrait alors (i)-(iii), et aussi (iv), (v) dans $\mathfrak{E}(s \frown i)$. Mais il se pourrait qu'il y ait plusieurs classes d'équivalence dans $\prod_{i \leq p} A_{i}$. On remarque alors que les conditions (i), (ii), (iv) et (v) sont héréditaires. La construction montrera qu'on peut procéder comme suit pour obtenir les conditions (iv) et (v) dans $\prod_{i \leq p} A_{i}$. Pour chaque couple $(s, t)$ de suites non équivalentes, on diminue les ouverts-fermés concernés (à savoir $U_{s}$ et $V_{t}$ pour la condition (iv), $\left(U_{s}\right.$ et $\left.U_{t}\right)$ ou $\left(V_{s}\right.$ et $\left.V_{t}\right)$ pour la condition $\left.(\mathrm{v})\right)$, 
de façon à assurer la condition ((iv) ou (v)) pour ce couple (c'est possible par rareté des graphes). Puis on assure la condition (iii), ce qui diminue les ouverts-fermés et permet de conserver les conditions (i) à (v) dans chaque classe. Comme les ensembles $A_{i}$ sont finis, on arrive ainsi à satisfaire les conditions (i) à (v) en un nombre fini d'étapes.

Montrons donc que cette nouvelle construction est possible. Si $n=1$, $\phi^{-1}(n)$ vaut $(0,1)$ et $z_{\phi^{-1}(n)}=s \frown i$; on choisit pour $U_{1}^{1}$ un ouvert-fermé de $\left(\bigcap_{q \leq p} O_{q}\right) \cap g_{0,0}^{-1}\left(V_{s}\right)$ de diamètre au plus $2^{-p-1}$ tel que $\delta\left(g_{0,0}\left[U_{1}^{1}\right]\right) \leq 2^{-p-1}$, et on pose $V_{1}^{1}:=g_{0,0}\left[U_{1}^{1}\right]$.

Admettons avoir construit les suites finies $U_{1}^{1}, V_{1}^{1}, \ldots, U_{1}^{n-1}, V_{1}^{n-1}$, $\ldots, U_{n-1}^{n-1}, V_{n-1}^{n-1}$, vérifiant (1)-(6), ce qui est fait pour $n=2$. On note $\mathfrak{T}(r, q):=\mathfrak{T}\left(z_{\phi^{-1}(r)}, z_{\phi^{-1}(q)}\right)$. La suite $z_{\phi^{-1}(n)}$ est dans $H_{\left(\phi^{-1}(n)\right)_{0}}$, donc on a $|\mathfrak{T}(1, n)|-1=\left(\phi^{-1}(n)\right)_{0}$. Comme $p_{0}=1,\left(\phi^{-1}(n)\right)_{0} \geq 1$, donc $|\mathfrak{T}(1, n)| \geq 2$ et $\mathfrak{T}(1, n)(|\mathfrak{T}(1, n)|-2) \in H_{\left(\phi^{-1}(n)\right)_{0}-1}$; par le choix de $\phi$, on peut trouver $m<n$ tel que $\mathfrak{T}(1, n)(|\mathfrak{T}(1, n)|-2)=z_{\phi^{-1}(m)}$. D'où $z_{\phi^{-1}(n)} \mathfrak{T} z_{\phi^{-1}(m)}$. Notons

$$
o:=\left\{\begin{array}{lll}
n\left(z_{\phi^{-1}(n)}, z_{\phi^{-1}(m)}\right) & \text { si } z_{\phi^{-1}(n)} \mathfrak{R} z_{\phi^{-1}(m)}, \\
n\left(z_{\phi^{-1}(m)}, z_{\phi^{-1}(n)}\right) & \text { si } z_{\phi^{-1}(m)} \mathfrak{R} z_{\phi^{-1}(n)} .
\end{array}\right.
$$

CAS $1: o<p$.

$1.1: z_{\phi^{-1}(m)} \Re z_{\phi^{-1}(n)}$. La suite $w(m, n)=\Phi\left(z_{\phi^{-1}(m)}\left\lceil(o+1), z_{\phi^{-1}(n)}\lceil(o+1))\right.\right.$ a déjà été définie et on a

$$
V_{z_{\phi^{-1}(n)\lceil p}}=g_{w(m, n)}\left[U_{z_{\phi^{-1}(m)\lceil p}}\right] .
$$

On choisit un ouvert-fermé $\widetilde{U}_{n}^{n}$ de $\left(\bigcap_{q<p} O_{q}\right) \cap g_{0,0}^{-1}\left(g_{w(m, n)}\left[U_{m}^{n-1}\right]\right)$ de diamètre au plus $2^{-p-1}$ tel que $\delta\left(g_{0,0}\left[\widetilde{U}_{n}^{n}\right]\right) \leq 2^{-p-1}$, on pose $\widetilde{V}_{n}^{n}:=g_{0,0}\left[\widetilde{U}_{n}^{n}\right]$, de sorte que les conditions (1) à (5) pour $k=l=n$ sont réalisées.

On définit ensuite les $\widetilde{U}_{q}^{n}$ et $\widetilde{V}_{q}^{n}$ pour $1 \leq q<n$, par récurrence sur $|\mathfrak{T}(q, n)|$. Comme $|\mathfrak{T}(q, n)| \geq 2, \widetilde{U}_{\mathfrak{T}(q, n)(1)}^{n}$ a été défini et il y a 2 cas. Soit $r$ entier compris entre 1 et $n$ tel que $\mathfrak{T}(q, n)(1)=z_{\phi^{-1}(r)}$ (la définition de $\phi$ montre l'existence de $r$ ).

1.1.1: Ou bien $z_{\phi^{-1}(r)} \mathfrak{R} z_{\phi^{-1}(q)}$, et on pose

$$
\widetilde{V}_{q}^{n}:=g_{w(r, q)}\left[\widetilde{U}_{r}^{n}\right], \quad \widetilde{U}_{q}^{n}:=g_{0,0}^{-1}\left(\widetilde{V}_{q}^{n}\right) .
$$

1.1.2 : Ou bien $z_{\phi^{-1}(q)} \mathfrak{R} z_{\phi^{-1}(r)}$, et on pose

$$
\widetilde{U}_{q}^{n}:=g_{w(q, r)}^{-1}\left(\widetilde{V}_{r}^{n}\right), \quad \widetilde{V}_{q}^{n}:=g_{0,0}\left[\widetilde{U}_{q}^{n}\right] .
$$

Montrons que ces définitions sont licites. On a $\mathfrak{T}(q, n)(1)=z_{\phi^{-1}(r)}$, où $1 \leq r \leq n$. Si le cas $r=n$ se produit, comme $z_{\phi^{-1}(m)}$ et $z_{\phi^{-1}(q)}$ sont dans $\mathfrak{E}(s \frown i)$, l'unicité de $\mathfrak{T}(1, n)$ montre que $q=m$. On en déduit que si $r=n$, 
on est dans le cas 1.1.2 puisqu'on ne peut pas avoir $z_{\phi^{-1}(q)}=z_{\phi^{-1}(r)}$, $\mathfrak{T}(q, n)$ étant sans répétition de termes $($ si $\widetilde{s} \mathfrak{R} \widetilde{t}$ et $\widetilde{t} \mathfrak{R} \widetilde{s}$, on a $\widetilde{s}=\widetilde{t})$.

Dans le cas 1.1.1, on a $r<n$ et $V_{q}^{n-1}=g_{w(r, q)}\left[U_{r}^{n-1}\right]$, donc $\widetilde{V}_{q}^{n}$ est un ouvert-fermé non vide de $V_{q}^{n-1}$, puisque $\widetilde{U}_{r}^{n} \subseteq U_{r}^{n-1}$. Par suite, $\widetilde{U}_{q}^{n}$ est un ouvert-fermé non vide de $U_{q}^{n-1}$. De même, $\widetilde{U}_{q}^{n}$ est un ouvert-fermé non vide de $U_{q}^{n-1}$ dans le cas 1.1.2, $r<n$. Si $r=n, q=m$ et la même conclusion vaut, par le choix de $\widetilde{U}_{n}^{n}$; par suite, $\widetilde{V}_{q}^{n}$ est un ouvert-fermé non vide de $V_{q}^{n-1}$. D'où la condition (6). Les conditions (1) et (2) pour $k=q$ en découlent. Vérifions (3). Soient donc $k, l \leq n$ tels que $z_{\phi^{-1}(k)} \Re z_{\phi^{-1}(l)}$. Si on a l'égalité $|\mathfrak{T}(k, n)|=|\mathfrak{T}(l, n)|=1$, on a $k=l=n$, et la condition (3) est réalisée (on utilise la condition (e)(i) d'une situation de départ). Plus généralement, la condition $(3)$ est réalisée si $k=l$. Si $|\mathfrak{T}(k, n)|=1$ et $|\mathfrak{T}(l, n)|=2$, la liaison entre $z_{\phi^{-1}(k)}$ et $z_{\phi^{-1}(l)}$ a déjà été prise en compte, par minimalité des longueurs. De même si $|\mathfrak{T}(k, n)|=2$ et $|\mathfrak{T}(l, n)|=1$. Si $|\mathfrak{T}(k, n)|,|\mathfrak{T}(l, n)| \geq 2$, on a $\mathfrak{T}(k, n)(1)=\mathfrak{T}(l, n)(0)$ ou bien alors $\mathfrak{T}(k, n)(0)=$ $\mathfrak{T}(l, n)(1)$, par unicité. Là encore, la liaison a été prise en compte. On a donc $\widetilde{V}_{l}^{n}=g_{w(k, l)}\left[\widetilde{U}_{k}^{n}\right]$. La condition (3) est donc réalisée, le seul nouveau cas étant celui où $r=n$ et $q=m$, et par la condition (e)(i) on a

$$
\begin{aligned}
w_{0}\left(z_{\phi^{-1}(m)}, z_{\phi^{-1}(n)}\right) & =w_{0}\left(z _ { \phi ^ { - 1 } ( m ) } \left\lceilp, z_{\phi^{-1}(n)}\lceil p) \geq \psi\left(z _ { \phi ^ { - 1 } ( m ) } \left\lceilp, z_{\phi^{-1}(n)}\lceil p)\right.\right.\right.\right. \\
& \geq \psi\left(z_{\phi^{-1}(m)}, z_{\phi^{-1}(n)}\right) .
\end{aligned}
$$

Remarquons que les conditions (1), (2), (4), (5), (6) sont héréditaires. Soient $k, l \in\{1, \ldots, n\}$ tels que $z_{\phi^{-1}(k)}<_{\text {lex }} z_{\phi^{-1}(l)}$ et $r \leq p$ tel que $\forall q \leq r$, $z_{\phi^{-1}(k)} \Re_{q} z_{\phi^{-1}(l)}$. On veut assurer que $\left(U_{k}^{n} \times V_{l}^{n}\right) \cap \bigcup_{q \leq r} \operatorname{Gr}\left(g_{q}\right)=\emptyset$. On a $\widetilde{V}_{l}^{n}=\widetilde{g}_{k, l}\left[\widetilde{U}_{k}^{n}\right]$, où $\widetilde{g}_{k, l}$ est de la forme $h h_{|\mathfrak{T}(k, l)|-2} \ldots h_{0}$ avec

$$
\begin{gathered}
h:= \begin{cases}\operatorname{Id}_{T} & \text { si } \operatorname{Im}\left(h_{|\mathfrak{T}(k, l)|-2}\right) \subseteq T, \\
g_{0,0} & \text { sinon, }\end{cases} \\
h_{0}:= \begin{cases}g_{w(\mathfrak{T}(k, l)(0), \mathfrak{T}(k, l)(1))} & \text { si } \mathfrak{T}(k, l)(0) \mathfrak{R} \mathfrak{T}(k, l)(1), \\
g_{w(\mathfrak{T}(k, l)(1), \mathfrak{T}(k, l)(0))}^{-1} g_{0,0} & \text { sinon, }\end{cases} \\
h_{p+1}:=\left\{\begin{array}{c}
g_{w(\mathfrak{T}(k, l)(p+1), \mathfrak{T}(k, l)(p+2))} \\
\operatorname{si} \mathfrak{T}(k, l)(p+1) \mathfrak{R} \mathfrak{T}(k, l)(p+2) \text { et } \operatorname{Im}\left(h_{p}\right) \subseteq Z, \\
g_{w(\mathfrak{T}(k, l)(p+2), \mathfrak{T}(k, l)(p+1))}^{-1} g_{0,0} \\
\operatorname{si} \mathfrak{T}(k, l)(p+2) \mathfrak{R} \mathfrak{T}(k, l)(p+1) \text { et } \operatorname{Im}\left(h_{p}\right) \subseteq Z, \\
g_{w(\mathfrak{T}(k, l)(p+1), \mathfrak{T}(k, l)(p+2))} g_{0,0}^{-1} \\
\operatorname{si} \mathfrak{T}(k, l)(p+1) \mathfrak{R} \mathfrak{T}(k, l)(p+2) \text { et } \operatorname{Im}\left(h_{p}\right) \subseteq T, \\
g_{w(\mathfrak{T}(k, l)(p+2), \mathfrak{T}(k, l)(p+1))}^{-1} \\
\operatorname{si} \mathfrak{T}(k, l)(p+2) \mathfrak{R} \mathfrak{T}(k, l)(p+1) \text { et } \operatorname{Im}\left(h_{p}\right) \subseteq T .
\end{array}\right.
\end{gathered}
$$


En renumérotant, on a donc $\widetilde{g}_{k, l}=g_{s_{2 r}} g_{s_{2 r-1}}^{-1} \ldots g_{s_{1}}^{-1} g_{s_{0}}$. On va donc chercher à assurer que

$$
\left(\widetilde{U}_{k}^{n} \times \widetilde{g}_{k, l}\left[\widetilde{U}_{k}^{n}\right]\right) \cap \bigcup_{q \leq r} \operatorname{Gr}\left(g_{q}\right)=\emptyset .
$$

Montrons par l'absurde que c'est possible. Comme les $g_{m, p}$ sont des homéomorphismes, $\widetilde{g}_{k, l}$ est définie et continue, donc on peut trouver un ouvert-fermé non vide $U \subseteq \widetilde{U}_{k}^{n}, q \leq r$ et $j \in \omega$ tels que pour tout $x \in U$, $g_{q}(x)=g_{q, j}(x)$ et $\widetilde{g}_{k, l}(x)=g_{q}(x)$. En d'autres termes, $g_{q, j}^{-1} \widetilde{g}_{k, l}(x)=x$. Comme $\left(Z, T,\left(g_{m, p}\right)_{(m, p) \in \omega^{2}}\right)$ est une situation d'arrivée, on a $s_{2 r}=(q, j)$ ou alors il existe $i<2 r$ tel que $s_{i}=s_{i+1}$. Montrons que cette seconde éventualité est exclue. Deux types de cas peuvent se produire :

$s_{i}=w(\mathfrak{T}(k, l)(j), \mathfrak{T}(k, l)(j+1)) \quad$ et $\quad s_{i+1}=w(\mathfrak{T}(k, l)(j+2), \mathfrak{T}(k, l)(j+1))$

$\mathrm{ou}$

$$
s_{i}=(0,0) \quad \text { et } \quad s_{i+1}=w(\mathfrak{T}(k, l)(j), \mathfrak{T}(k, l)(j+1)) .
$$

Comme $\Phi$ est injective, $\mathfrak{T}(k, l)(j)\lceil(n(\mathfrak{T}(k, l)(j), \mathfrak{T}(k, l)(j+1))+1)$ vaut $\mathfrak{T}(k, l)(j+2)\lceil(n(\mathfrak{T}(k, l)(j+2), \mathfrak{T}(k, l)(j+1))+1)$ et aussi

$$
n(\mathfrak{T}(k, l)(j), \mathfrak{T}(k, l)(j+1))+1=n(\mathfrak{T}(k, l)(j+2), \mathfrak{T}(k, l)(j+1))+1 .
$$

Par définition de $n(s, t)$, on a $\mathfrak{T}(k, l)(j)=\mathfrak{T}(k, l)(j+2)$, ce qui contredit la définition de $\mathfrak{T}(k, l)$.

Dans l'autre type de cas, l'injectivité de $\Phi$ et la définition de $n(s, t)$ font que $\mathfrak{T}(k, l)(j)=\mathfrak{T}(k, l)(j+1)$, ce qui est également absurde.

On a donc $s_{2 r}=(q, j), r=0$ et $z_{\phi^{-1}(k)} \mathfrak{R} z_{\phi^{-1}(l)}$; d'autre part, l'entier $w_{0}(\mathfrak{T}(k, l)(0), \mathfrak{T}(k, l)(1))=q \leq r$, et $\psi\left(z_{\phi^{-1}(k)}, z_{\phi^{-1}(l)}\right) \leq w_{0}\left(z_{\phi^{-1}(k)}, z_{\phi^{-1}(l)}\right)$ $\leq r$; d'où $z_{\phi^{-1}(k)} \mathfrak{R}_{\psi\left(z_{\phi^{-1}(k)}, z_{\phi^{-1}(l)}\right)} z_{\phi^{-1}(l)}$, avec $\psi\left(z_{\phi^{-1}(k)}, z_{\phi^{-1}(l)}\right) \leq r$, ce qui est absurde.

En diminuant $\widetilde{U}_{k}^{n}$ et en assurant la condition (3), on peut donc avoir la condition (4) pour le couple $(k, l)$. Comme ces couples sont en nombre fini, on obtient la condition (4) en un nombre fini d'étapes.

On doit maintenant assurer (5). Il existe une relation du type $\widetilde{U}_{l}^{n}=$ $\widetilde{g}_{k, l}\left[\widetilde{U}_{k}^{n}\right]$. On montre alors comme avant qu'on peut diminuer $\widetilde{U}_{k}^{n}$ de façon à assurer la disjonction de $\widetilde{U}_{k}^{n}$ et $\widetilde{U}_{l}^{n}$. De même, il existe une relation du type $\widetilde{V}_{l}^{n}=\widetilde{g}_{k, l}\left[\widetilde{V}_{k}^{n}\right]$. Là encore, on peut diminuer $\widetilde{V}_{k}^{n}$ de façon à assurer la disjonction de $\widetilde{V}_{k}^{n}$ et $\widetilde{V}_{l}^{n}$. En effet, on a $\widetilde{g}_{k, l}^{\prime}(x)=x$ pour tout $x$ d'un ouvert-fermé non vide de $Z$, où $\widetilde{g}_{k, l}^{\prime}:=g_{(0,0)}^{-1} \widetilde{g}_{k, l} g_{(0,0)}$. On raisonne alors comme dans le cas précédent pour avoir une contradiction.

$1.2: z_{\phi^{-1}(n)} \mathfrak{R} z_{\phi^{-1}(m)}$. Ce cas est analogue au précédent (on a $V_{z_{\phi^{-1}(m)\lceil p}}$ $=g_{w(n, m)}\left[U_{z_{\phi^{-1}(n)\lceil p}}\right]$, on choisit $\widetilde{U}_{n}^{n}$ dans $g_{w(n, m)}^{-1}\left(V_{m}^{n-1}\right) \cap \bigcap_{q \leq p} O_{q}$, et seul le cas 1.2.1 est possible si $r=n$ ). 
CAS 2: $o=p$.

$2.1: z_{\phi^{-1}(m)} \mathfrak{R} z_{\phi^{-1}(n)}$. Dans ce cas $n\left(z_{\phi^{-1}(m)}\left\lceil p, z_{\phi^{-1}(n)}\lceil p)<o=p\right.\right.$. On a $V_{z_{\phi^{-1}(n)}\lceil p}=g_{w\left(z_{\phi^{-1}(m)}\left\lceil p, z_{\phi^{-1}(n)}\lceil p)\right.\right.}\left[U_{z_{\phi^{-1}(m)}\lceil p}\right]$, et $\bigcup_{m \in \omega} \operatorname{Gr}\left(g_{m}\right)$ ren-

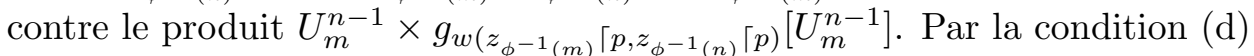
d'une situation générale, on peut trouver $q \geq \psi\left(z_{\phi^{-1}(m)}, z_{\phi^{-1}(n)}\right)$ et $j \in \omega$ tels que $\left(U_{m}^{n-1} \times g_{w\left(z_{\phi^{-1}(m)}\left\lceil p, z_{\phi^{-1}(n)}\lceil p)\right.\right.}\left[U_{m}^{n-1}\right]\right) \cap \operatorname{Gr}\left(g_{q, j}\right) \neq \emptyset$. On pose $\Phi\left(z_{\phi^{-1}(m)}, z_{\phi^{-1}(n)}\right):=(q, j)$, en ayant pris soin de choisir $q$ suffisamment grand pour assurer l'injectivité de $\Phi$.

L'ensemble ci-dessous est un ouvert non vide :

$$
\left(\bigcap_{q \leq p} O_{q}\right) \cap g_{(0,0)}^{-1}\left(g_{w(m, n)}\left[U_{m}^{n-1} \cap g_{w(m, n)}^{-1}\left(g_{w\left(z _ { \phi ^ { - 1 } ( m ) } \left\lceilp, z_{\phi^{-1}(n)}\lceil p)\right.\right.}\left[U_{m}^{n-1}\right]\right)\right]\right) .
$$

On choisit $\widetilde{U}_{n}^{n}$ dans cet ouvert et on raisonne comme en 1.1.1.

$2.2: z_{\phi^{-1}(n)} \mathfrak{R} z_{\phi^{-1}(m)}$. On choisit $\widetilde{U}_{n}^{n}$ dans l'intersection ci-dessous :

$$
g_{q, j}^{-1}\left(V_{m}^{n-1}\right) \cap g_{w\left(z _ { \phi ^ { - 1 } ( n ) } \left\lceilp, z_{\phi^{-1}(m)}\lceil p)\right.\right.}^{-1}\left(V_{m}^{n-1}\right) \cap \bigcap_{q \leq p} O_{q} .
$$

Puis on raisonne comme en 2.1 , en posant $\Phi\left(z_{\phi^{-1}(n)}, z_{\phi^{-1}(m)}\right):=(q, j)$.

Le lemme 6 de la section 3 assurera l'existence d'une situation de départ. Le reste de cette section est consacré à la recherche, dans chaque borélien $A$ dont toutes les coupes sont dénombrables et n'étant pas $\operatorname{pot}\left(\boldsymbol{\Pi}_{2}^{0}\right)$, d'une réunion $\bigcup_{m \in \omega} \operatorname{Gr}\left(g_{m}\lceil G(g))\right.$ se réduisant à $A$, où $\left(Z, T,\left(g_{m, p}\right)_{(m, p) \in \omega^{2}}\right)$ est une situation d'arrivée. Pour ce faire, il se trouve qu'un résultat intermédiaire (le théorème 3) va être utilisé deux fois. Pour l'énoncer, il nous faut une définition supplémentaire.

DÉfinition. On dira que $\left(Z, T,\left(h_{n, p}\right)_{(n, p) \in \omega^{2}}, M\right)$ est un système réducteur si :

(a) $Z$ et $T$ sont des espaces polonais parfaits de dimension 0 non vides.

(b) $h_{n, p}$ est un homéomorphisme de domaine (respectivement d'image) ouvert-fermé de $Z$ (respectivement de $T$ ), de diamètre au plus $2^{-\Delta(n, p)}$, où $\Delta: \omega^{2} \rightarrow \omega$ est injective.

(c) $M$ est $G_{\delta}$ de $Z \times T$ de projection dense dans $Z$, et disjoint de $\bigcup_{(n, p) \in \omega^{2}} \operatorname{Gr}\left(h_{n, p}\right)$.

(d) Pour tout ouvert $O$ de $Z \times T$ tel que $\Pi_{Z}[M \cap O]$ soit dense dans $Z$, $\Pi_{Z}[M \cap O]$ est comaigre dans $Z$.

(e) Si $U$ et $V$ sont ouverts-fermés et $M$ rencontre $U \times V$, alors $\operatorname{Gr}\left(h_{n, p}\right) \cap \bar{M} \cap(U \times V)$ est le graphe de la restriction de $h_{n, p}$ à un ouvert $U_{n, p, U, V}$ de $U$ et $\left\{(n, p) \in \omega^{2} \mid \operatorname{Gr}\left(h_{n, p}\right) \cap \bar{M} \cap(U \times V) \neq \emptyset\right\}$ est infini. 
Avec le lemme 2 et le théorème 3 qui suivent, nous reprenons pour l'essentiel la preuve du théorème 2.11 de [Le2]; seul le vocabulaire change. Le lemme 2 donne un procédé pour obtenir des systèmes réducteurs. Le lemme 5 nous en fournira un autre.

Lemme 2. Soient $X, Y$ des espaces polonais, $A$ un borélien de $X \times Y$ dont les coupes horizontales et verticales sont dénombrables n'étant pas $\operatorname{pot}\left(\boldsymbol{\Pi}_{2}^{0}\right)$. Alors il existe un système réducteur $\left(Z, T,\left(h_{n, p}\right)_{(n, p) \in \omega^{2}}, M\right)$ et des injections continues $u: Z \rightarrow X$ et $v: T \rightarrow Y$ tels que :

(a) $\bigcup_{(n, p) \in \omega^{2}} \operatorname{Gr}\left(h_{n, p}\right) \subseteq(u \times v)^{-1}(A)$.

(b) $M \subseteq(u \times v)^{-1}(\check{A})$.

Démonstration. On peut supposer, pour simplifier l'écriture, que $X$ et $Y$ sont récursivement présentés, et que $A$ est $\Delta_{1}^{1}$-réunion de graphes $\Delta_{1}^{1}$ de fonctions injectives. En effet, $A$ est réunion dénombrable de graphes de fonctions boréliennes, $A$ étant à coupes verticales dénombrables (cf. $[\mathrm{Ke}]$ ). $A$ étant à coupes horizontales dénombrables, ces fonctions sont countable-toone; on applique alors le lemme 2.4.a de [Le2] pour voir que $A$ est réunion dénombrable de graphes d'injections boréliennes. Désignons par $W^{X}$ un ensemble $\Pi_{1}^{1}$ dans $\omega$ de codes pour les $\Delta_{1}^{1}$ de $X$, et par $C^{X} \subseteq \omega \times X$ un ensemble $\Pi_{1}^{1}$ dont les sections aux points de $W^{X}$ décrivent les $\Delta_{1}^{1}$ de $X$, et tel que la relation " $n \in W^{X}$ et $(n, x) \notin C^{X}$ " soit $\Pi_{1}^{1}$ (cf. [Lo1]). Soit également $W \subseteq W^{X \times Y}$ un ensemble $\Pi_{1}^{1}$ de codes pour les $\Delta_{1}^{1} \cap \operatorname{pot}\left(\boldsymbol{\Sigma}_{2}^{0}\right)$ de $X \times Y$ (dont l'existence est démontrée dans [Lo1]).

Posons

$$
H:=\bigcup\left\{(E \times F) \backslash A \mid E, F \in \Delta_{1}^{1} \text { et }(E \times F) \backslash A \in \operatorname{pot}\left(\boldsymbol{\Sigma}_{2}^{0}\right)\right\} .
$$

On a

$$
\begin{aligned}
H(x, y) \Leftrightarrow & \exists n \in W \exists m(m)_{0} \in W^{X} \text { et }(m)_{1} \in W^{Y} \text { et } \forall z \forall t \\
& {\left[( n \in W ^ { X \times Y } \text { et } ( n , z , t ) \notin C ^ { X \times Y } ) \text { ou } \left\{\left((m)_{0}, z\right) \in C^{X}\right.\right. \text { et }} \\
& \left.\left.\left((m)_{1}, t\right) \in C^{Y} \text { et }(z, t) \notin A\right\}\right] \text { et } \\
& {\left[\left((m)_{0} \in W^{X} \text { et }\left((m)_{0}, z\right) \notin C^{X}\right)\right. \text { ou }} \\
& \left((m)_{1} \in W^{Y} \text { et }\left((m)_{1}, t\right) \notin C^{Y}\right) \text { ou } \\
& \left.(z, t) \in A \text { ou }(n, z, t) \in C^{X \times Y}\right] \\
& \text { et }\left((m)_{0}, x\right) \in C^{X} \text { et }\left((m)_{1}, y\right) \in C^{Y} \text { et }(x, y) \notin A .
\end{aligned}
$$

Donc $H$ est $\Pi_{1}^{1}$. Posons $N:=\check{A} \cap \check{H} ; N$ est $\Sigma_{1}^{1}$ et $G_{\delta}$ de $X \times Y$ muni de la topologie $\Delta_{X} \times \Delta_{Y}\left(\Delta_{X}\right.$ est la topologie engendrée par les $\Delta_{1}^{1}$ de $X)$. En effet, comme $A$ est $\operatorname{pot}\left(\boldsymbol{\Sigma}_{2}^{0}\right)$ et $\Delta_{1}^{1}, A$ est $\boldsymbol{\Sigma}_{2}^{0}$ pour la topologie $\Delta_{X} \times \Delta_{Y}$ (voir [Lo1]). De même, $H$ est réunion dénombrable de $\boldsymbol{\Sigma}_{2}^{0}$ pour $\Delta_{X} \times \Delta_{Y}$. Posons $D_{X}:=\left\{x \in X \mid x \notin \Delta_{1}^{1}\right\}, \Omega_{X}:=\left\{x \in X \mid \omega_{1}^{x}=\omega_{1}^{\mathrm{CK}}\right\}$, et $Z_{0}:=\Omega_{X} \cap D_{X}, T:=\Omega_{Y} \cap D_{Y}$. On munit $Z_{0}$ (respectivement $T$ ) de la 
restriction de la topologie $\Sigma_{X}$ (respectivement $\Sigma_{Y}$ ) de GandyHarrington sur $X$ (respectivement $Y$ ), de sorte que $Z_{0}$ et $T$ sont polonais parfaits de dimension 0 . En effet, les traces des $\Sigma_{1}^{1}$ sur $\Omega_{X}$ sont ouvertsfermés de $\left(\Omega_{X}, \Sigma_{X}\left\lceil\Omega_{X}\right)\right.$ : si $B$ est $\Sigma_{1}^{1}$ contenu dans $\Omega_{X}$ et $f$ est $\Delta_{1}^{1}$ telle que $f(x) \in \mathrm{WO} \Leftrightarrow x \notin B$, on a

$$
x \notin B \Leftrightarrow x \notin \Omega_{X} \text { ou }\left(x \in \Omega_{X} \text { et } \exists \xi<\omega_{1}^{\mathrm{CK}} \quad(f(x) \in \text { WO et }|f(x)| \leq \xi)\right) \text {. }
$$

L'espace $\left(\Omega_{X}, \Sigma_{X}\left\lceil\Omega_{X}\right)\right.$ est donc à base dénombrable d'ouverts-fermés, donc métrisable séparable ; on sait (cf. [HKL] et [Mo]) que c'est un espace fortement $\alpha$-favorable, comme ouvert (puisque $\Sigma_{1}^{1}$ ) d'un espace fortement $\alpha$-favorable. C'est donc un espace polonais (cf. [Ke]), de dimension 0 par ce qui précède.

On pose $M:=\left(Z_{0} \times T\right) \cap N, Z:=\Pi_{Z_{0}}[M]$. L'ensemble $M$ est non vide. En effet, $N$ rencontre $D_{X} \times D_{Y}$, sinon $N$ serait $\operatorname{pot}\left(\Delta_{1}^{0}\right)$ et $A \cup H$ aussi, donc $A=(A \cup H) \cap \check{H}$ serait $\operatorname{pot}\left(\boldsymbol{\Pi}_{2}^{0}\right)$, ce qui est exclus. Donc $N \cap\left(D_{X} \times D_{Y}\right)$, qui est $\Sigma_{1}^{1}$, rencontre $\Omega_{X \times Y}$, par le théorème de base de Gandy (cf. [Lo3]). Comme $\Omega_{X \times Y} \subseteq \Omega_{X} \times \Omega_{Y}, M \neq \emptyset$. Donc $Z$ et $T$ sont non vides et on a la condition (a) d'un système réducteur, puisque $Z$ est $\Sigma_{1}^{1}$, donc ouvert-fermé de $Z_{0}$. De plus, $M$ est $G_{\delta}$ de $Z \times T$ et $\Pi_{Z}[M]=Z$ est dense dans $Z$.

Si $O$ est ouvert de $Z \times T$, il est réunion de rectangles $\Sigma_{1}^{1}$, donc $\Pi_{Z}[M \cap O]$ est ouvert de $Z$; par suite, si $\Pi_{Z}[M \cap O]$ est dense dans $Z, \Pi_{Z}[M \cap O]$ est comaigre dans $Z$. D'où la condition $(\mathrm{d})$ d'un système réducteur.

Posons $A=\bigcup_{n \in \omega}$, disj. $\operatorname{Gr}\left(g_{n}\right)$, où $g_{n}$ est un homéomorphisme de domaine et d'image ouverts-fermés non vides (en fait $\Delta_{1}^{1}$; le raffinement des topologies permet de rendre les bijections boréliennes bicontinues). Soient $U$ et $V$ tels que $M \cap(U \times V) \neq \emptyset$, ouverts-fermés. Pour voir que $\{n \in \omega \mid$ $\left.\operatorname{Gr}\left(g_{n}\right) \cap \bar{M} \cap(U \times V) \neq \emptyset\right\}$ est infini, on peut supposer que $U$ et $V$ sont $\Sigma_{1}^{1}$. Admettons avoir trouvé $n_{1}, \ldots, n_{r}$ deux à deux distincts tels que $\operatorname{Gr}\left(g_{n_{i}}\right) \cap$ $\bar{M} \cap(U \times V) \neq \emptyset$ pour $0<i \leq r$. Posons $A^{\prime}:=\bigcup_{n \notin\left\{n_{1}, \ldots, n_{r}\right\}} \operatorname{Gr}\left(g_{n}\right) ; A^{\prime}$ est $\Delta_{1}^{1}$, et $A=A^{\prime} \cup \bigcup_{0<i \leq r} \operatorname{Gr}\left(g_{n_{i}}\right)$. Posons $\mathcal{O}:=N \cap(U \times V)(\mathcal{O}=M \cap(U \times V)$ car $\left.U \times V \subseteq Z \times T \subseteq Z_{0} \times T\right)$. Supposons que $A^{\prime} \cap \overline{\mathcal{O}}^{\Sigma_{X} \times \Sigma_{Y}}=\emptyset$. Alors, par double application du théorème de séparation, $A^{\prime} \cap \overline{\mathcal{O}}^{\Delta_{X} \times \Delta_{Y}}=\emptyset$, donc on a la triple inclusion

$$
\begin{aligned}
(U \times V) \backslash A^{\prime} & \subseteq \mathcal{O} \cup H \cup \bigcup_{0<i \leq r} \operatorname{Gr}\left(g_{n_{i}}\right) \\
& \subseteq \overline{\mathcal{O}}^{\Delta_{X} \times \Delta_{Y}} \cup H \cup \bigcup_{0<i \leq r} \operatorname{Gr}\left(g_{n_{i}}\right) \subseteq \check{A}^{\prime} .
\end{aligned}
$$

Donc $(U \times V) \backslash A^{\prime}$ et $A^{\prime}$ sont deux $\Sigma_{1}^{1}$ séparables par un $\operatorname{pot}\left(\boldsymbol{\Sigma}_{2}^{0}\right)$; ils peuvent par conséquent être séparés par un ensemble $K \in \Delta_{1}^{1} \cap \operatorname{pot}\left(\boldsymbol{\Sigma}_{2}^{0}\right)$ (cf. [Lo1]). On a $U \times V \subseteq K \cup A^{\prime}$, donc on peut trouver $\mathcal{U}$ et $\mathcal{V}$, deux $\Delta_{1}^{1}$ tels que 
$U \times V \subseteq \mathcal{U} \times \mathcal{V} \subseteq K \cup A^{\prime}$. D'où l'on a $(\mathcal{U} \times \mathcal{V}) \backslash A^{\prime}=K \cap(\mathcal{U} \times \mathcal{V})$ et $(\mathcal{U} \times \mathcal{V}) \backslash A=K \cap(\mathcal{U} \times \mathcal{V}) \cap\left[\bigcap_{0<i<r}(\mathcal{U} \times \mathcal{V}) \backslash \operatorname{Gr}\left(g_{n_{i}}\right)\right]$ est $\operatorname{pot}\left(\boldsymbol{\Sigma}_{2}^{0}\right)$. Donc $(\mathcal{U} \times \mathcal{V}) \backslash A \subseteq H$, puis $\mathcal{O} \subseteq H \backslash H=\emptyset$, ce qui est absurde. Par conséquent, $A^{\prime} \cap \overline{\mathcal{O}}^{\bar{\Sigma}_{X} \times \Sigma_{Y}} \neq \emptyset$. Or $\mathcal{O} \subseteq D_{X} \times D_{Y}$, donc $\overline{\mathcal{O}}^{\Sigma_{X} \times \Sigma_{Y}} \subseteq$ $D_{X} \times D_{Y}$. Donc $\left(D_{X} \times D_{Y}\right) \cap A^{\prime} \cap \overline{\mathcal{O}}^{\Sigma_{X} \times \bar{\Sigma}_{Y}} \neq \emptyset$ et est $\Sigma_{1}^{1}$, donc rencontre $\Omega_{X \times Y} \subseteq \Omega_{X} \times \Omega_{Y}$, donc $\left(Z_{0} \cap T\right) \cap A^{\prime} \cap \overline{\mathcal{O}}^{\Sigma_{X} \times \Sigma_{Y}} \neq \emptyset$. Comme $U$ est ouvert-fermé de $Z_{0}, A^{\prime} \cap \overline{\mathcal{O}}^{Z \times T} \neq \emptyset$. L'existence d'un ouvert $U_{n, U, V}$ de $U$ tel que $\operatorname{Gr}\left(g_{n}\left\lceil U_{n, U, V}\right)=\operatorname{Gr}\left(g_{n}\right) \cap \bar{M} \cap(U \times V)\right.$ vient du fait que $\bar{M}$ est $\Sigma_{1}^{1}$.

Soit $\Delta: \omega^{2} \rightarrow \omega$ injective. On construit des ouverts-fermés $D_{n, p}$ de $Z$ tels que :

- $D_{n}:=\bigcup_{p \in \omega} D_{n, p}$ soit dense dans $D_{g_{n}} \cap Z \cap g_{n}^{-1}(T)$.

- $\delta\left(D_{n, p}\right), \delta\left(g_{n}\left[D_{n, p}\right]\right) \leq 2^{-\Delta(n, p)}$.

Soit $\left(x_{p}^{n}\right)_{p}$ une suite dense de $D_{g_{n}} \cap Z \cap g_{n}^{-1}(T)$. On choisit un ouvertfermé $D_{n, p}$ de $D_{g_{n}} \cap Z \cap g_{n}^{-1}(T)$, contenant $x_{p}^{n}$, de diamètre au plus $2^{-\Delta(n, p)}$, dont l'image par $g_{n}$ soit aussi de diamètre au plus $2^{-\Delta(n, p)}$. Il est clair que $D_{n, p}$ convient.

Il reste à poser $h_{n, p}:=g_{n}\left\lceil D_{n, p}\right.$. On a $U_{n, p, U, V}=U_{n, U, V} \cap D_{n, p}$. Enfin, on a vu qu'il y a une infinité d'entiers $n$ tels que $\operatorname{Gr}\left(g_{n}\right) \cap \bar{M} \cap(U \times V) \neq \emptyset$ si $M \cap(U \times V) \neq \emptyset$, et on a un ouvert non vide $U_{n, U, V}$ de $D_{n}^{\emptyset}$. Par densité, on trouve $p$ tel que $U_{n, U, V} \cap D_{n, p} \neq \emptyset$. Par suite, on a une infinité de couples $(n, p)$ tels que $\operatorname{Gr}\left(h_{n, p}\right) \cap \bar{M} \cap(U \times V) \neq \emptyset$. Il reste à poser $u:=\mathrm{Id}: Z \rightarrow X$ et $v:=\operatorname{Id}: T \rightarrow Y$.

A partir d'un système réducteur, on n'obtient pas tout de suite une situation d'arrivée, mais seulement une situation générale dans un premier temps :

ThÉorème 3. Soit $\left(Z, T,\left(k_{q, p}\right)_{(q, p) \in \omega^{2}}, N\right)$ un système réducteur. Alors il existe une injection $\Phi: \omega^{2} \rightarrow \omega^{2}$ et des ouverts-fermés $D_{m, p} \subseteq D_{k_{\Phi(m, p)}}$ tels que si $g_{m, p}:=k_{\Phi(m, p)}\left\lceil D_{m, p}\right.$ :

(a) $\left(Z, T,\left(g_{m, p}\right)_{(m, p) \in \omega^{2}}\right)$ soit une situation générale.

(b) Pour $x$ dans $G(g)$ et $y$ dans $\overline{g[x]} \backslash g[x],(x, y)$ est dans $N$.

Si de plus $\left(Z, T,\left(k_{q, p}\right)_{(q, p) \in \omega^{2}}\right)$ est une situation d'arrivée, on peut avoir $G(g) \subseteq G(k)$ et $\left(Z, T,\left(g_{m, p}\right)_{(m, p) \in \omega^{2}}\right)$ d'arrivée.

Démonstration. Soit

$$
\nu:\left\{\begin{aligned}
\omega^{<\omega} & \rightarrow \omega, \\
s & \mapsto \begin{cases}0 & \text { si } s=\emptyset \\
\sum_{i<|s|}(s(i)+1) & \text { sinon. }\end{cases}
\end{aligned}\right.
$$


On choisit une distance $d^{\prime} \leq 1$ complète sur $N$. On note, pour $x \in Z, d_{x}^{\prime}$ la distance complète sur $N^{x}$ définie par la formule $d_{x}^{\prime}(y, t):=d^{\prime}((x, y),(x, t))$. Pour exprimer l'absence de points isolés dans $g[x]$, il est plus commode d'indexer les fonctions par $\omega^{<\omega} \times \omega$ que par $\omega^{2}$. Soient donc $e: \omega \rightarrow \omega^{<\omega}$ bijective, $\psi:=e \times \operatorname{Id}_{\omega}$ et $\theta:=e^{-1} \times \operatorname{Id}_{\omega}$. On va en fait construire une injection $\phi: \omega^{<\omega} \times \omega \rightarrow \omega^{2}$, et on posera ensuite $\Phi:=\phi \circ \psi$. On va également construire, par récurrence sur $|t|$, où $t \in \omega^{<\omega}$ :

- Des ouverts-fermés $D_{t, p}$ de $D_{k_{\phi(t, p)}}$.

- Des $G_{\delta}$ denses $\mathcal{G}_{t}$ de $D_{t}:=\bigcup_{p \in \omega} D_{t, p}$.

- Des ouverts à coupes verticales ouvertes-fermées $\omega_{t}$ de $Z \times T$.

- Des ouverts $G_{t}$ de $N$.

On demande à ces objets de vérifier :

(1) $\bar{D}_{t}=Z$.

(2) $D_{t, p} \cap D_{t, q}=\emptyset$ si $p \neq q$.

(3) $\forall x \in D_{t \frown n} \cap \mathcal{G}_{t} \quad d\left(k_{t}(x), k_{t \frown n}(x)\right) \leq 2^{1-\nu\left(t^{\frown} n\right)}$, où $k_{t}$ est le recollement des fonctions $k_{\phi(t, p)}\left\lceil D_{t, p}\right.$, pour $p$ entier.

(4) $\operatorname{Gr}\left(k_{t}\right) \subseteq \omega_{t} \cap \bar{G}_{t}$.

(5) $\omega_{t \frown n} \subseteq \omega_{t}$ et $\omega_{t \frown n} \cap \omega_{t \frown m}=\emptyset$ si $n \neq m$.

(6) $\forall x \in Z \quad \delta\left(\omega_{t}^{x}\right) \leq 2^{-\nu(t)}$.

(7) $\forall x \in Z \quad \overline{G_{t-n}^{x}} \cap N^{x} \subseteq G_{t}^{x} \subseteq \omega_{t}^{x}$, et $\mathcal{G}_{t} \subseteq \Pi_{Z}\left[G_{t}\right]$.

(8) $\forall x \in Z \quad \delta_{x}^{\prime}\left(G_{t}^{x}\right) \leq 2^{-|t|}$

Admettons ceci réalisé. On pose $D_{m, p}:=D_{\psi(m, p)}, G(g):=\bigcap_{t \in \omega<\omega} \mathcal{G}_{t}$. Les conditions (a) et (b) d'une situation générale sont clairement réalisées. On a $D_{g_{m, p}}=D_{m, p}=D_{\psi(m, p)}$, donc $\bigcup_{p \in \omega} D_{g_{m, p}}=\bigcup_{p \in \omega} D_{\psi(m, p)}$ vaut $\bigcup_{p \in \omega} D_{e(m), p}=D_{e(m)}$. L'ouvert $\bigcup_{p \in \omega} D_{g_{m, p}}$ est donc dense dans $Z$, par (1). De plus, la réunion est disjointe, par (2). D'où la condition (c) d'une situation générale. On a $G(g)=\bigcap_{t \in \omega<\omega} \mathcal{G}_{t}$, donc $G(g)$ est $G_{\delta}$ dense de $Z$, et si $x \in G(g)$ et $m \in \omega, x \in \mathcal{G}_{e(m)} \subseteq D_{e(m)}=\bigcup_{p \in \omega} D_{g_{m, p}}=D_{g_{m}}$. Donc $G(g) \subseteq \bigcap_{m \in \omega} D_{g_{m}}$. Par (3) et la remarque qui suit, on a la condition (d) d'une situation générale.

Notons que $g[x]=\left\{k_{t}(x) \mid t \in \omega^{<\omega}\right\}$. En effet, si $t \in \omega^{<\omega}$, il existe $p \in \omega$ tel que $k_{t}(x)=k_{\phi(t, p)}(x)=k_{\Phi(\theta(t, p))}(x)=g_{\theta(t, p)}(x)=g_{e^{-1}(t)}(x)$. Inversement, si $m \in \omega$, on trouve $p \in \omega$ tel que $g_{m}(x)=g_{m, p}(x)$ soit égal à $k_{\Phi(m, p)}(x)=k_{e(m)}(x)$.

L'argument qui suit est semblable à celui utilisé dans la preuve du théorème d'Hurewicz dans [SR]. On pose $M_{k}:=\left\{k_{t}(x)|| t \mid \leq k\right\}$. Alors $M_{k}$ est fermé dans $T$, par récurrence sur $k$ : si $M_{k}^{\varepsilon}:=\left\{y \in T \mid d\left(y, M_{k}\right) \leq \varepsilon\right\}$, on a $M_{k+1}=\bigcap_{\varepsilon>0}\left[M_{k}^{\varepsilon} \cup\left(M_{k+1} \backslash M_{k}^{\varepsilon}\right)\right]$, et $M_{k+1} \backslash M_{k}^{\varepsilon}$ est fini, par (3).

Si $k \in \omega$ et $y \in \overline{g[x]} \backslash g[x]$, alors $y \notin M_{k}$. Donc il existe $\varepsilon>0$ tel que $y \notin M_{k}^{\varepsilon}$. Si $t \in \omega^{k}$ et $(x, w)$ est dans $\omega_{t}$, on a 


$$
d\left(w, M_{k}\right) \leq d\left(w, k_{t}(x)\right) \leq \delta_{x}\left(\omega_{t}^{x}\right) \leq 2^{-\nu(t)} \leq \varepsilon
$$

dès que $\nu(t) \geq k_{0}$, donc $\omega_{t}^{x} \subseteq M_{k}^{\varepsilon}$, sauf pour un nombre fini de $t$ dans $\omega^{k}$. Donc si on définit $\mathcal{H}:=\left\{t \in \omega^{k} \mid \omega_{t}^{x} \nsubseteq M_{k}^{\varepsilon}\right\}$, on a la suite d'inclusions suivante :

$$
\begin{aligned}
g[x] & =M_{k} \cup\left\{k_{t}(x)|| t \mid>k\right\} \subseteq M_{k} \cup \bigcup_{|w|>k} \omega_{w}^{x} \\
& \subseteq M_{k} \cup \bigcup_{|t|=k} \omega_{t}^{x} \subseteq M_{k}^{\varepsilon} \cup \bigcup_{t \in \mathcal{H}} \omega_{t}^{x} .
\end{aligned}
$$

D'où $\overline{g[x]} \subseteq M_{k}^{\varepsilon} \cup \bigcup_{t \in \mathcal{H}} \omega_{t}^{x} \subseteq M_{k}^{\varepsilon} \cup \bigcup_{|t|=k} \omega_{t}^{x}$. Donc on trouve une unique suite $\sigma$ dans $\omega^{\omega}$ telle que $y \in \bigcap_{t \prec \sigma} \omega_{t}^{x}$. Puisque $\left(\overline{G_{t}^{x}} \cap N^{x}\right)_{t \prec \sigma}$ est une suite décroissante de fermés non vides dont les diamètres tendent vers 0 de $\left(N^{x}, d_{x}^{\prime}\right)$, par (7) et (8), elle converge vers $\xi \in N^{x}$, et $\{\xi\}=\bigcap_{t \prec \sigma} G_{t}^{x}$. D'où $\xi \in \bigcap_{t \prec \sigma} \omega_{t}^{x}=\{y\}$ et $(x, y) \in N$.

Montrons donc que la construction est possible. Soit $\left(Z_{n}\right)$ une base de la topologie de $Z$ formée d'ouverts-fermés non vides. Comme $Z$ est polonais de dimension 0 , il peut être vu comme un fermé de $\omega^{\omega}$; on peut donc supposer que $Z$ est muni d'une distance complète telle que $d\left(Z_{n}, \check{Z}_{n}\right)>0$. On pose $\omega_{\emptyset}:=Z \times T, G_{\emptyset}:=N$. On construit $\phi(\emptyset, p)$ et $D_{\emptyset, p}$ par récurrence sur $p$, en exigeant de plus que $\bigcup_{q<p} D_{\emptyset, q} \neq Z$. Admettons avoir construit $\phi(\emptyset, q)$ et $D_{\emptyset, q}$ pour $q<p$. On peut définir, pour $q \leq p$,

$$
n(q):=\min \left\{n \in \omega \mid Z_{n} \cap \bigcup_{r<q} D_{\emptyset, r}=\emptyset \text { et } \forall r<q \quad n>n(r)\right\} .
$$

Comme $\Pi_{Z}[N]$ est dense dans $Z$, on a $N \cap\left(Z_{n(p)} \times T\right) \neq \emptyset$, donc par la condition (e) d'un système réducteur, on peut trouver $(m, r) \in \omega^{2} \backslash\{\phi(\emptyset, q) \mid$ $q<p\}$ tel que $\delta\left(D_{k_{m, r}}\right)<d\left(Z_{n(p)}, \check{Z}_{n(p)}\right)$ et $\operatorname{Gr}\left(k_{m, r}\right) \cap \bar{N} \cap\left(Z_{n(p)} \times T\right) \neq \emptyset$. On a donc $D_{k_{m, r}} \subseteq Z_{n(p)}$, et on pose $\phi(\emptyset, p):=(m, r)$; on choisit $D_{\emptyset, p} \subseteq$ $U_{m, r, Z_{n(p)}, T}$ tel que $\bigcup_{q \leq p} D_{\emptyset, q} \neq Z$, de sorte que la condition (1) est réalisée. Ceci termine la construction pour $|t|=0$.

On effectue maintenant une sous-construction : on construit, par récurrence sur $n$ :

- Les ouverts $\omega_{t}{ }_{n}$.

- Une suite décroissante $\left(E_{n}\right)$ de $G_{\delta}$ denses de $D_{t}$.

- Des fonctions continues $f_{n}: E_{n} \rightarrow T$.

- Des ouverts $V_{n}$ de $Z \times T$.

On demande à ces objets de vérifier :

(i) $\operatorname{Gr}\left(f_{n}\right) \subseteq \omega_{t \frown n} \cap G_{t}$.

(ii) $\omega_{t \frown n} \subseteq \omega_{t}$ et $\omega_{t \frown n} \cap \omega_{t \frown m}=\emptyset$ si $n \neq m$.

(iii) $\forall x \in E_{n} \quad d\left(f_{n}(x), k_{t}(x)\right) \leq 2^{-\nu\left(t^{\frown} n\right)}$.

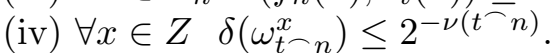

(v) $\operatorname{Gr}\left(k_{t}\left\lceil E_{n}\right) \subseteq V_{n} \subseteq(Z \times T) \backslash \bigcup_{m \leq n} \omega_{t \frown m}\right.$. 
Montrons qu'une telle construction est possible. Si on a construit ces objets pour $q<n$, soit $x \in D_{t}$. Par continuité de $k_{t}$, on peut trouver un voisinage ouvert $\mathcal{W}_{x}$ de $x$ inclus dans $D_{t}$ et un voisinage ouvert-fermé $\mathcal{V}_{x}$ de diamètre au plus $2^{-\nu\left(t^{\frown} n\right)}$ de $k_{t}(x)$ tels que $\mathcal{W}_{x} \subseteq k_{t}^{-1}\left(\mathcal{V}_{x}\right)$, de sorte que si $(z, t) \in \mathcal{W}_{x} \times \mathcal{V}_{x}$, alors $d\left(t, k_{t}(z)\right) \leq 2^{-\nu\left(t^{\frown} n\right)}$. On a, par la propriété de Lindelöf, $\bigcup_{x \in D_{t}}\left(\mathcal{W}_{x} \times \mathcal{V}_{x}\right)=\bigcup_{m \in \omega}\left(\mathcal{W}_{m} \times \mathcal{V}_{m}\right)$; on réduit la suite $\left(\mathcal{W}_{m}\right)$ en $\left(\mathcal{W}_{m}^{\prime}\right)$, puis on pose $\mathcal{U}_{n}:=\bigcup_{m \in \omega}\left(\mathcal{W}_{m}^{\prime} \times \mathcal{V}_{m}\right)$. Soit $\mathcal{O}_{n}$ un ouvert dense de $D_{t}$ contenant $E_{n-1}$ tel que $\operatorname{Gr}\left(k_{t}\right) \cap V_{n-1}=\operatorname{Gr}\left(k_{t}\left\lceil\mathcal{O}_{n}\right)\right.$. Par le théorème de Jankov-von Neumann, on peut trouver $\widetilde{f}_{n}$ Baire-mesurable uniformisant $\mathcal{U}_{n} \cap G_{t}$ sur sa projection $\Pi$. Or $\Pi$ est dense dans $Z$. En effet, soient $U$ un ouvert-fermé non vide de $Z$ et $x \in \mathcal{O}_{n} \cap U$. Comme $\left(x, k_{t}(x)\right) \in \mathcal{U}_{n} \cap \bar{G}_{t}$, on peut trouver un voisinage ouvert-fermé $W$ de $x$ tel que $W \times k_{t}[W] \subseteq$ $\mathcal{U}_{n} \cap(U \times T)$. Soit alors $(z, y) \in\left(W \times k_{t}[W]\right) \cap G_{t}$. On a $z \in U \cap \Pi \neq \emptyset$. Par la condition (d) d'un système réducteur, $\Pi$ est comaigre dans $Z$, donc contient un $G_{\delta}$ dense $W_{n}$ de $Z$. Alors $\widetilde{f}_{n}\left\lceil W_{n}\right.$ est Baire-mesurable, donc on peut trouver un $G_{\delta}$ dense $F_{n}$ de $W_{n} \cap D_{t}$ tel que $\widetilde{f}_{n}\left\lceil F_{n}\right.$ soit continue. On peut poser $E_{n}:=F_{n} \cap E_{n-1}$ et $f_{n}:=\widetilde{f}_{n}\left\lceil E_{n}\right.$. Si $x \in E_{n}$, on a $\left(x, f_{n}(x)\right) \in \mathcal{U}_{n}$, donc $d\left(f_{n}(x), k_{t}(x)\right) \leq 2^{-\nu\left(t^{\frown} n\right)}$. Les graphes de $f_{n}$ et $k_{t}\left\lceil E_{n}\right.$ sont des fermés disjoints de $E_{n} \times T$, donc on peut trouver un ouvert-fermé $\Theta$ de $E_{n} \times T$ tel que $\operatorname{Gr}\left(f_{n}\right) \subseteq \Theta \subseteq\left(E_{n} \times T\right) \backslash \operatorname{Gr}\left(k_{t}\left\lceil E_{n}\right)\right.$.

On peut trouver des ouverts disjoints $\mathcal{T}$ et $\mathcal{W}$ de $Z \times T$ tels que l'on a $\Theta=\left(E_{n} \times T\right) \cap \mathcal{T}$ et $\left(E_{n} \times T\right) \backslash \Theta=\left(E_{n} \times T\right) \cap \mathcal{W}$, par la propriété de réduction des ouverts. Soit $\left(T_{m}\right)$ une base de la topologie de $T$ stable par intersections finies et formée d'ouverts-fermés vérifiant $d\left(T_{m}, \check{T}_{m}\right)>0$. On raisonne alors comme précédemment : pour $x$ dans $E_{n}$, on trouve un voisinage ouvertfermé de base $\mathcal{V}_{x}^{\prime}$ de $f_{n}(x)$ de diamètre au plus $2^{-\nu\left(t^{\frown} n\right)}$ et un voisinage ouvert-fermé $\mathcal{Y}_{x}$ de $x$ tels que $\mathcal{Y}_{x} \cap E_{n} \subseteq f_{n}^{-1}\left(\mathcal{V}_{x}^{\prime}\right)$ et $\mathcal{Y}_{x} \times \mathcal{V}_{x}^{\prime} \subseteq \mathcal{T} \cap V_{n-1} \cap \omega_{t}$. Comme avant, on applique la propriété de Lindelöf, ce qui fournit $\mathcal{Y}_{m}$ et $\mathcal{V}_{m}^{\prime}$, et on réduit la suite $\left(\mathcal{Y}_{m}\right)$ en $\left(\mathcal{Y}_{m}^{\prime}\right)$. On pose $\omega_{t \frown n}:=\bigcup_{m \in \omega} \mathcal{Y}_{m}^{\prime} \times \mathcal{V}_{m}^{\prime}$ et $V_{n}:=V_{n-1} \cap \mathcal{W}$. Les conditions (i) à (v) sont clairement satisfaites. On a donc les conditions (5) et (6) de la construction principale.

On procède encore comme avant pour définir $G_{t}{ }_{n}$. Pour $x$ dans $E_{n}$, on trouve un voisinage ouvert-fermé de base $\mathcal{D}_{x}$ de $f_{n}(x)$ et un voisinage ouvertfermé $\mathcal{C}_{x}$ de $x$ tels que $\delta^{\prime}\left(\left[\mathcal{C}_{x} \times \mathcal{D}_{x}\right] \cap N\right) \leq 2^{-|t|-1}, \mathcal{C}_{x} \cap E_{n} \subseteq f_{n}^{-1}\left(\mathcal{D}_{x}\right)$ et $\mathcal{C}_{x} \times \mathcal{D}_{x} \subseteq \omega_{t} \frown n \cap G \cap\left(D_{t} \times T\right)$, où $G$ est ouvert de $Z \times T$ avec $G \cap N=$ $G_{t}$. On applique la propriété de Lindelöf, ce qui fournit $\mathcal{C}_{m}$ et $\mathcal{D}_{m}$, et on réduit la suite $\left(\mathcal{C}_{m}\right)$ en $\left(\mathcal{C}_{m}^{\prime}\right)$. On pose $G_{t \frown n}:=N \cap\left(\bigcup_{m \in \omega} \mathcal{C}_{m}^{\prime} \times \mathcal{D}_{m}\right)$ et $\mathcal{G}_{t}:=\bigcap_{n \in \omega} E_{n}$, et les conditions (7) et (8) sont satisfaites. On a la suite d'égalités

$G_{t \frown n} \cap \omega_{t \frown n}=N \cap\left(\bigcup_{m \in \omega} \mathcal{C}_{m}^{\prime} \times \mathcal{D}_{m}\right) \cap\left(\bigcup_{m \in \omega} \mathcal{Y}_{m}^{\prime} \times \mathcal{V}_{m}^{\prime}\right)=\bigcup_{l \in \omega} N \cap\left(A_{l} \times B_{l}\right)$ 
(avec par définition $A_{l}:=\mathcal{C}_{e_{2}^{-1}(l)_{0}}^{\prime} \cap \mathcal{Y}_{e_{2}^{-1}(l)_{1}}^{\prime}$ et $B_{l}:=\mathcal{D}_{e_{2}^{-1}(l)_{0}} \cap \mathcal{V}_{e_{2}^{-1}(l)_{1}}^{\prime}$, où $e_{2}: \omega^{2} \rightarrow \omega$ est bijective). De plus, on a $A_{l} \cap A_{l^{\prime}}=\emptyset$ si $l \neq l^{\prime}$ et $\Pi_{Z}\left[N \cap\left(A_{l} \times B_{l}\right)\right]$ est comaigre dans $A_{l}$ car $\operatorname{Gr}\left(f_{n}\right) \subseteq G_{t \frown n} \cap \omega_{t \frown n}$. On procède alors comme pour $t=\emptyset$ pour terminer la construction, en travaillant dans $A_{l} \times B_{l}$. On construit, par récurrence sur $p, \phi\left(t^{\frown} n, e_{2}(l, p)\right)$ et $D_{t \frown n, e_{2}(l, p)}$, en exigeant que $\bigcup_{q<p} D_{t \frown n, e_{2}(l, q)} \neq A_{l}$. On travaille avec la base $\left(Z_{n_{k}}\right)_{k}$ de $A_{l}$ formée des $Z_{n}$ inclus dans $A_{l}$. On pose

$$
k(q):=\min \left\{k \in \omega \mid Z_{n_{k}} \cap \bigcup_{r<q} D_{t \frown n, e_{2}(l, r)}=\emptyset \text { et } \forall r<q \quad k>k(r)\right\} .
$$

On choisit $(m, r) \in \omega^{2} \backslash\left\{\phi\left(t^{\frown} n, e_{2}(l, q)\right) \mid q<p\right\}$ de façon à avoir les inégalités $\delta\left(D_{k_{m, r}}\right)<d\left(Z_{n_{k(p)}}, \check{Z}_{n_{k(p)}}\right), \delta\left(\operatorname{Im}\left(k_{m, r}\right)\right)<d\left(B_{l}, \check{B}_{l}\right)$, et aussi $\operatorname{Gr}\left(k_{m, r}\right) \cap \bar{N} \cap\left(Z_{n_{k(p)}} \times B_{l}\right) \neq \emptyset$. On pose $\phi\left(t \frown n, e_{2}(l, p)\right):=(m, r)$ et on choisit $D_{t \frown n, e_{2}(l, p)}$ dans $U_{m, r, Z_{n k(p)}, B_{l}}$ tel que $\overline{\bigcup_{q \leq p} D_{t \frown n, e_{2}(l, q)}} \neq A_{l}$. Ceci termine la construction car $\operatorname{Gr}\left(k_{m, r}\right) \subseteq Z_{n_{k(p)}} \times B_{l} \subseteq \omega_{t \frown n}$, ce qui assure l'injectivité de $\phi$.

Si de plus $\left(Z, T,\left(k_{q, p}\right)_{(q, p) \in \omega^{2}}\right)$ est d'arrivée, quitte à remplacer $G(g)$ par $G(g) \cap G(k)$, on peut avoir $G(g) \subseteq G(k)$. L'injectivité de $\Phi$ fait que la situation générale $\left(Z, T,\left(g_{m, p}\right)_{(m, p) \in \omega^{2}}\right)$ est en fait d'arrivée.

On doit maintenant obtenir une situation d'arrivée à partir d'une situation générale :

ThÉORÈme 4. Soit $\left(Z, T,\left(l_{r, p}\right)_{(r, p) \in \omega^{2}}\right)$ une situation générale. Alors il existe une situation d'arrivée $\left(Z, T,\left(k_{q, p}\right)_{(q, p) \in \omega^{2}}\right)$ telle que $G(k) \subseteq G(l)$ et pour $x$ dans $G(k)$, on ait $k[x] \subseteq l[x]$.

Démonstration. Pour exprimer à la fois l'absence de points isolés dans $k[x]$, la densité de $D_{k_{q}}$ dans $Z$ et la condition (c) d'une situation d'arrivée, il est plus commode d'indexer les fonctions par $\bigcup_{n \in \omega} \omega^{n} \times \omega^{n+1}$ que par $\omega^{2}$. Soient donc $\psi: \operatorname{Im}(M) \rightarrow \omega$ bijective croissante, et $\phi:=\psi \circ M$, où

$$
M:\left\{\begin{aligned}
\bigcup_{n \in \omega} \omega^{n} \times \omega^{n+1} & \rightarrow \omega, \\
(s, t) & \mapsto q_{0}^{t(0)+1} q_{1}^{s(0)+1} \ldots q_{2 n-2}^{t(n-1)+1} q_{2 n-1}^{s(n-1)+1} q_{2 n}^{t(n)+1}
\end{aligned}\right.
$$

$\left(\left(q_{n}\right)\right.$ est la suite des nombres premiers). Donc $\phi$ est une bijection de $\bigcup_{n \in \omega} \omega^{n} \times \omega^{n+1}$ sur $\omega$ vérifiant

$$
\phi(s, t)<\phi\left(s \frown p, t^{\frown} m\right) \text { et } \phi(s, t)<\phi(s, t\lceil|s| \frown[t(|s|)+1])
$$

pour $(s, t)$ dans $\bigcup_{n \in \omega} \omega^{n} \times \omega^{n+1}$ et $(p, m)$ dans $\omega^{2}$. Soient $\left(Z_{n}\right)$ une base de la topologie de $Z$, et $\left(O_{r}\right)$ une suite d'ouverts denses de $Z$ tels que l'on a $G(l)=\bigcap_{r \in \omega} O_{r}$. En supposant

$$
\forall n \in \omega \forall s, t \in \omega^{n} \forall m \in \omega \quad D_{k_{s\lceil(|s|-1), t}} \backslash \bigcup_{i<m} D_{k_{s, t}{ }_{i}} \neq \emptyset
$$


où $D_{k_{\emptyset\lceil-1, \emptyset}}:=Z$, on définit l'application $r$ de $\bigcup_{n \geq-1} \omega^{n} \times \omega^{n+1}$ dans $\omega \cup\{-1\}$ en posant

$$
\begin{aligned}
& r(\emptyset\lceil-1, \emptyset):=-1 \text {, } \\
& r\left(s, t^{\frown} m\right):=\min \left\{r \in \omega \mid Z_{r} \cap D_{k_{s\lceil(|s|-1), t}} \backslash \bigcup_{i<m} D_{k_{s, t}{ }_{i}} \neq \emptyset,\right. \\
& r>\max \left(r\left(s\lceil(|s|-1), t), \max _{i<m} r\left(s, t^{\frown} i\right)\right)\right\} .
\end{aligned}
$$

On a donc $r\left(s, t^{\frown}(n+1)\right)>r(s, t \frown n)>r(s\lceil(|s|-1), t)$.

On construit des fonctions $k_{s, t \frown m}$, pour $(s, t, m) \in\left(\bigcup_{n \in \omega}\left(\omega^{n}\right)^{2}\right) \times \omega$, par récurrence sur $\phi\left(s, t^{\frown} m\right)$, en demandant :

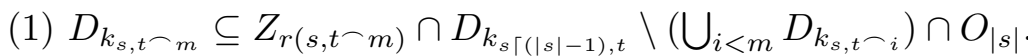

(2) $\bigcup_{i<m} D_{k_{s, t} \frown{ }_{i}} \neq D_{k_{s \Gamma(|s|-1), t}}$ et $D_{k_{s\lceil(|s|-1), t}} \neq \emptyset$.

(3) $\delta\left(D_{k_{s, t} \frown m}\right), \delta\left(\operatorname{Im}\left(k_{s, t \frown m}\right)\right) \leq 2^{-\phi\left(s, t^{\frown} m\right)}$.

(4) $k_{s, t \frown m}$ est la restriction d'une des $l_{r, p}$ à un ouvert-fermé de $D_{l_{r, p}}$.

(5) $\forall n, m \in \omega \forall s, t \in \omega^{n} \forall x \in D_{k_{s, t}{ }_{m}}$

$$
d\left(k_{s, t \succ m}(x), k_{s\lceil(|s|-1), t}(x)\right)<2^{-s(|s|-1)} .
$$

(6) $\forall h \in \omega \backslash\{0\} \forall u \in\left(\bigcup_{p \leq \phi(s, t \frown m)}\left\{\phi^{-1}(p)\right\}\right)^{2 h}$

$$
\begin{aligned}
\left(\exists U \in \Delta_{1}^{0}, \emptyset \neq U \subseteq Z \forall x \in U \quad k_{u(0)}^{-1} k_{u(1)}\right. & \left.\ldots k_{u(2 k-2)}^{-1} k_{u(2 k-1)}(x)=x\right) \\
& \Rightarrow(\exists i<2 h-1 \quad u(i)=u(i+1)) .
\end{aligned}
$$

(7) Il n'y a qu'un nombre fini de compositions du type de la condition (6) sans termes consécutifs identiques ayant un domaine de définition non vide.

Admettons cette construction réalisée. Soient $e: \omega \rightarrow \omega^{<\omega}$ et, pour $n>0, e_{n}: \omega^{n} \rightarrow \omega$ bijectives. On pose $k_{q, p}:=k_{e(q), e_{|e(q)|+1}^{-1}(p)}$. Notons que les applications suivantes sont réciproques l'une de l'autre :

$$
\begin{aligned}
\omega^{2} & \rightarrow \bigcup_{n \in \omega} \omega^{n} \times \omega^{n+1}, & \bigcup_{n \in \omega} \omega^{n} \times \omega^{n+1} & \rightarrow \omega^{2}, \\
(q, p) & \mapsto\left(e(q), e_{|e(q)|+1}^{-1}(p)\right), & \left(s, t^{\frown} m\right) & \mapsto\left(e^{-1}(s), e_{|s|+1}\left(t^{\frown} m\right)\right) .
\end{aligned}
$$

Les conditions (a) et (b) d'une situation générale et les conditions (b) et (c) d'une situation d'arrivée seront alors clairement réalisées, par (3), (4) et (6). De plus $\bigcup_{m \in \omega} D_{k_{s, t}{ }_{m}}$ est dense dans $D_{k_{s\lceil(|s|-1), t}}$, sinon on peut trouver $r>r\left(s\lceil(|s|-1), t)\right.$ tel que $Z_{r} \subseteq D_{k_{s\lceil(|s|-1), t}} \backslash \overline{\bigcup_{m \in \omega} D_{k_{s, t} \succ_{m}}}$. Comme la suite $(r(s, t \frown m))_{m}$ croît strictement vers l'infini, on trouve un plus petit $m$ tel que $r(s, t \frown m)>r$.

Si $m>0$, on a $r\left(s, t^{\frown}(m-1)\right) \leq r$; si $r\left(s, t^{\frown}(m-1)\right)<r$, on a $r(s, t \frown m) \leq r$, ce qui est absurde. Si $r=r(s, t \frown(m-1)), D_{k_{s, t} \frown(m-1)} \subseteq Z_{r}$, ce qui contredit la disjonction de $Z_{r}$ et $D_{k_{s, t} \frown(m-1)}$. Si $m=0$, on a $r\left(s, t^{-} 0\right)>r>r(s\lceil(|s|-1), t)$, ce qui contredit la définition de $r(s, t \frown 0)$. 
Il suffit donc d'assurer (1) et (2) pour avoir la disjonction de $D_{k_{s, t}{ }_{m}}$ et $D_{k_{s, t}{ }_{n}}$ pour $n \neq m$, donc de $D_{k_{s, t} \frown_{m}}$ et $D_{k_{s, v}{ }_{n}}$ pour $(t, m) \neq(v, n)$, et la densité de $D_{s}:=\bigcup_{(t, m) \in \omega^{|s|} \times \omega} D_{k_{s, t}{ }_{m}}$ dans $\bigcup_{t \in \omega^{|s|} \mid} D_{k_{s\lceil(|s|-1), t}} ;$ on a donc la condition (c) d'une situation générale. Enfin, on pose $G(k):=\bigcap_{q \in \omega} D_{k_{q}}$ et la condition (5) entraîne la condition (d) d'une situation générale car si $q \in \omega$ et $x \in G(k)=\bigcap_{s \in \omega<\omega} D_{s}, x \in D_{e(q)} \cap D_{e(q) \frown p}$ pour tout entier $p$, donc il existe $t \in \omega^{|e(q)|+1}$ et $m \in \omega$ tels que $x \in D_{e(q) \frown p, t \frown m} \subseteq D_{e(q), t}$, et $d\left(k_{q}(x), k_{l}(x)\right)<2^{-p}$, où $e(l)=e(q) \frown p$.

Montrons donc que cette construction est possible. Soit $(r, p) \in \omega^{2}$ telle que $D_{l_{r, p}} \cap Z_{0} \neq \emptyset$. On choisit un ouvert-fermé non vide $D_{k_{\phi^{-1}(0)}}$ strictement inclus dans $D_{l_{r, p}} \cap Z_{0} \cap O_{\left|\phi_{0}^{-1}(0)\right|}$ et on pose $k_{\phi^{-1}(0)}:=l_{r, p}\left\lceil D_{k_{\phi^{-1}(0)}}\right.$. Admettons avoir construit $\left(k_{s, t \succ m}\right)_{\phi(s, t \smile m) \leq n}$ vérifiant (1)-(7), ce qui est fait pour $n=0$. Posons $\phi^{-1}(n+1):=\left(s, t^{\frown} m\right)$. On a déjà construit $D_{k_{\phi^{-1}\left(q_{1}\right)}}$, $\ldots, D_{k_{\phi^{-1}\left(q_{m}\right)}}$ dans $D_{k_{s\lceil(|s|-1), t}}$, de sorte que $\phi^{-1}\left(q_{i}\right)=(s, t \frown(i-1))$ pour $1 \leq i \leq m$, par construction de $\phi$.

Soit $U$ un ouvert-fermé non vide de $D_{k_{s\lceil(|s|-1), t}}$ tel que

$$
\delta(U), \delta\left(k_{s\lceil(|s|-1), t}[U]\right) \leq 2^{-n-1}, \quad U \cup \bigcup_{i<m} D_{k_{s, t} \frown_{i}} \neq D_{k_{s\lceil(|s|-1), t}},
$$

et tel que $U \times k_{s\lceil(|s|-1), t}[U]$ soit inclus dans

$$
\begin{aligned}
&\left\{(x, y) \in\left[Z_{r(s, t \smile m)} \cap D_{k_{s\lceil(|s|-1), t} \backslash} \backslash \bigcup_{i<m} D_{k_{s, t}{ }_{i}}\right] \times T \mid\right. \\
&\left.d\left(y, k_{s\lceil(|s|-1), t}(x)\right)<2^{-s(|s|-1)}\right\} .
\end{aligned}
$$

Ce qui suit est à rapprocher du lemme 2.11 de [Le3]. Par (7), on note $\left\{H_{1}, \ldots, H_{p}\right\}$ l'ensemble fini des compositions du type de la condition (6) sans termes consécutifs identiques de domaine de définition non vide définissables à partir de $k_{\phi^{-1}(0)}, \ldots, k_{\phi^{-1}(n)}$. Pour $I \subseteq p$, on pose $O_{I}:=$ $\bigcap_{i \in I} D_{H_{i+1}}$

$\cap \bigcap_{i \in p \backslash I} \check{D}_{H_{i+1}}$. Alors $\left(O_{I}\right)_{I \subseteq p}$ est une partition en ouverts-fermés de $Z$, donc on trouve $I \subseteq p$ tel que $U \cap O_{I} \neq \emptyset$. Soit

$$
O^{\prime}:=\left\{x \in U \cap O_{I} \mid \forall i \in I \quad H_{i+1}(x) \neq x\right\} .
$$

$\operatorname{Par}(6), O^{\prime}$ est un ouvert dense de $U \cap O_{I}$; on peut donc trouver un ouvertfermé non vide $O^{\prime \prime}$ de $O^{\prime}$ tel que pour tout $i \in I, O^{\prime \prime} \cap H_{i+1}\left[O^{\prime \prime}\right]=\emptyset$. Soit $x \in O^{\prime \prime} \cap G(l)$, et $(r, p) \in \omega^{2}$ telle que $\left(x, l_{r, p}(x)\right) \in O^{\prime \prime} \times k_{s\lceil(|s|-1), t}\left[O^{\prime \prime}\right]$, $l_{r, p}(x) \neq k_{\phi^{-1}(j)} H_{m+1}(x)$ et aussi $l_{r, p}(x) \neq k_{\phi^{-1}(j)}(x)$ pour tout $m<p$ et tout $j \leq n$. On choisit $D_{k_{\phi^{-1}(n+1)}}$ contenant $x$ dans l'ouvert $D_{l_{r, p}} \cap O^{\prime \prime} \cap$ $l_{r, p}^{-1}\left(k_{s\lceil(|s|-1), t}\left[O^{\prime \prime}\right]\right) \cap O_{\left|\phi_{0}^{-1}(n+1)\right|}$ tel que pour tout $m<p$ et pour tout $j \leq n$ on ait $l_{r, p}\left[D_{k_{\phi^{-1}(n+1)}}\right] \cap k_{\phi^{-1}(j)} H_{m+1}\left[D_{k_{\phi^{-1}(n+1)}}\right]=\emptyset$ et $l_{r, p}\left[D_{k_{\phi^{-1}(n+1)}}\right] \cap$ $k_{\phi^{-1}(j)}\left[D_{k_{\phi^{-1}(n+1)}}\right]=\emptyset$. On pose $k_{\phi^{-1}(n+1)}:=l_{r, p}\left\lceil D_{k_{\phi^{-1}(n+1)}}\right.$. 
Il est clair que les conditions (1) à (5) sont réalisées. Montrons que la condition (6) est réalisée au rang $n+1$, en raisonnant par l'absurde. On peut donc trouver $h>0, u \in\left(\bigcup_{p \leq n+1}\left\{\phi^{-1}(p)\right\}\right)^{2 h}$ et un ouvert-fermé non vide $U$ de $Z$ tels que pour tout $x$ de $U$ on ait $k_{u(0)}^{-1} k_{u(1)} \ldots k_{u(2 h-2)}^{-1} k_{u(2 h-1)}(x)=x$ et $u(i) \neq u(i+1)$ si $i<2 h-1$. Par hypothèse de récurrence, on peut trouver $i<2 h$ minimal tel que $u(i)=\phi^{-1}(n+1)$. Montrons, en raisonnant par l'absurde, qu'un tel $i$ est unique. Si tel n'est pas le cas, on peut trouver $j>i+1$ minimal tel que $u(j)=\phi^{-1}(n+1)$. Il y a alors quatre cas.

CAS $1: i$ et $j$ sont impairs. Posons $y:=k_{u(j)} k_{u(j+1)}^{-1} \ldots k_{u(2 h-1)}(x)$. Comme $u(j)=\phi^{-1}(n+1), y$ est dans $k_{s\lceil(|s|-1), t}\left[O^{\prime \prime}\right]$ et on trouve $z$ dans $O^{\prime \prime}$ tel que $y=k_{s\lceil(|s|-1), t}(z)$. On a

$$
x=k_{u(0)}^{-1} k_{u(1)} \ldots k_{u(j-1)}^{-1}(y)=k_{u(0)}^{-1} k_{u(1)} \ldots k_{u(j-1)}^{-1} k_{s\lceil(|s|-1), t}(z),
$$

donc le domaine définition de $k_{u(i+1)}^{-1} k_{u(i+2)} \ldots k_{u(j-1)}^{-1} k_{s\lceil(|s|-1), t}$ est non vide. Si $i+1=j-1$ et $u(j-1)=s\left\lceil(|s|-1), t\right.$, on a $z \in D_{k_{\phi}-1(n+1)}$ et $y$ est dans $k_{\phi^{-1}(n+1)}\left[D_{k_{\phi^{-1}(n+1)}}\right] \cap k_{s\lceil(|s|-1), t}\left[D_{k_{\phi^{-1}(n+1)}}\right]=\emptyset$. $\stackrel{\mathrm{Si}}{{ }^{n}}$ $i+1 \neq j-1$ ou $u(j-1) \neq s\lceil(|s|-1), t$, on peut trouver $r<p$ tel que $H_{r+1}=k_{u(i+1)}^{-1} k_{u(i+2)} \ldots k_{u(j-1)}^{-1} k_{s\lceil(|s|-1), t}$. Comme $z \in O^{\prime \prime} \subseteq O_{I}$, $H_{r+1}(z) \notin O^{\prime \prime}$, donc $k_{u(i)}\left(H_{r+1}(z)\right)$ n'est pas défini, ce qui est absurde.

CAS 2 : $i$ et $j$ sont pairs. Dans la composition apparaît

$$
k_{\phi^{-1}(n+1)}^{-1} k_{u(i+1)} \ldots k_{u(j-1)} k_{\phi^{-1}(n+1)}^{-1},
$$

donc la composition $k_{\phi^{-1}(n+1)} k_{u(j-1)}^{-1} \ldots k_{u(i+1)}^{-1} k_{\phi^{-1}(n+1)}$ a un domaine de définition non vide. Mais on voit comme avant que c'est impossible.

CAS $3: i$ est impair et $j$ est pair. Soit $r<p$ tel que

$$
H_{r+1}=k_{u(i+1)}^{-1} \ldots k_{u(j-1)} .
$$

Alors $k_{u(j)}^{-1} \ldots k_{u(2 h-1)}(x) \in O^{\prime \prime}$, donc comme avant $k_{u(i+1)}^{-1} \ldots k_{u(2 h-1)}(x)$ n'est pas dans $O^{\prime \prime}$, ce qui est absurde.

CAS $4: i$ est pair et $j$ est impair. Posons $y:=k_{u(j)} \ldots k_{u(2 h-1)}(x)$; comme dans le cas 1 , on trouve $z$ dans $O^{\prime \prime}$ tel que $y=k_{s\lceil(|s|-1), t}(z)$. Posons $w:=k_{u(i+2)}^{-1} \ldots k_{u(2 h-1)}(x) ;$ on a $w=k_{u(i+2)}^{-1} \ldots k_{u(j-1)}^{-1} k_{s\lceil(|s|-1), t}(z)$, et $k_{u(i+1)}(w)=k_{s\lceil(|s|-1), t}(v)$, où $v \in O^{\prime \prime}$, puisque $k_{\phi^{-1}(n+1)}^{-1}\left(k_{u(i+1)}(w)\right)$ est défini. On a $v=k_{s\lceil(|s|-1), t}^{-1} k_{u(i+1)} \ldots k_{u(j-1)}^{-1} k_{s\lceil(|s|-1), t}(z)$. D'où $u(i+1)=$ $s\lceil(|s|-1), t=u(j-1)$ et donc $i+2<j-1$, ce qui est absurde (on utilise le fait que $z$ et $v$ sont dans $\left.O^{\prime \prime}\right)$.

L'entier $i$ est donc unique et on peut trouver un ouvert-fermé non vide de $D_{k_{\phi^{-1}(n+1)}}$ sur lequel $k_{\phi^{-1}(n+1)}$ coïncide avec une composition des fonctions 
$k_{\phi^{-1}(0)}, \ldots, k_{\phi^{-1}(n)}$ de la forme $k_{\phi^{-1}(j)} H_{m+1}$, où $m<p$. Mais ceci est contraire à la construction de $k_{\phi^{-1}(n+1)}$. Pour vérifier la condition (7), on remarque que dans une composition du type de la condition (6) sans termes consécutifs identiques des fonctions $k_{\phi^{-1}(0)}, \ldots, k_{\phi^{-1}(n+1)}$, il y a au plus une fois la fonction $k_{\phi^{-1}(n+1)}$ (comme précédemment). Une telle composition est donc nécessairement de la forme

$$
\begin{aligned}
& H_{m+1}, \quad H_{m+1} k_{\phi^{-1}(i)}^{-1} k_{\phi^{-1}(n+1)}, \quad H_{m+1} k_{\phi^{-1}(n+1)}^{-1} k_{\phi^{-1}(i)}, \\
& H_{m+1} k_{\phi^{-1}(i)}^{-1} k_{\phi^{-1}(n+1)} H_{m^{\prime}+1}, \quad H_{m+1} k_{\phi^{-1}(n+1)}^{-1} k_{\phi^{-1}(i)} H_{m^{\prime}+1} \text {, } \\
& k_{\phi^{-1}(i)}^{-1} k_{\phi^{-1}(n+1)} H_{m+1} \quad \text { ou } \quad k_{\phi^{-1}(n+1)}^{-1} k_{\phi^{-1}(i)} H_{m+1} \text {, }
\end{aligned}
$$

où $m, m^{\prime}<p$ et $i \leq n$. Il n'y en a donc qu'un nombre fini.

Il reste à pouvoir assurer la réduction de la situation d'arrivée au borélien dont nous sommes partis. Le lemme qui suit, couplé avec le théorème 3 , va le permettre.

LEMME 5. On suppose que $\left(Z, T,\left(l_{r, p}\right)_{(r, p) \in \omega^{2}}\right)$ est une situation générale et que $\left(Z, T,\left(k_{q, p}\right)_{\left.(q, p) \in \omega^{2}\right)}\right.$ est une situation d'arrivée telles que pour tout $x$ de $G(k) \cap G(l), k[x] \subseteq l[x]$. Alors il existe un ensemble $N, G_{\delta}$ de $(Z \times T) \backslash \bigcup_{r \in \omega} \operatorname{Gr}\left(l_{r}\right)$, tel que $\left(Z, T,\left(k_{q, p}\right)_{(q, p) \in \omega^{2}}, N\right)$ soit un système réducteur.

Démonstration. Posons $H:=G(k) \cap G(l)$ et aussi

$$
N:=\{(x, y) \in Z \times T \mid x \in H \text { et } y \in \overline{k[x]} \backslash l[x]\} .
$$

Alors $N$ est clairement $G_{\delta}$, et on a $N \cap \bigcup_{r \in \omega} \operatorname{Gr}\left(l_{r}\right)=\emptyset$, ainsi que les conditions (a) et (b) d'un système réducteur.

Soit $U$ un ouvert-fermé non vide de $Z$. On choisit $x \in H \cap U$. Comme $\left(Z, T,\left(k_{q, p}\right)_{(q, p) \in \omega^{2}}\right)$ est une situation d'arrivée, $k[x]$ est dénombrable sans point isolé, donc on trouve $y$ dans $\overline{k[x]} \backslash l[x]$, puisque $\overline{k[x]}$ est polonais parfait et que $l[x]$ est dénombrable. On a alors $x \in U \cap \Pi_{Z}[N]$ puisque $(x, y) \in N$. D'où la condition (c) d'un système réducteur.

Soient $x \in U \cap \Pi_{Z}[N \cap O], y \in \overline{k[x]} \cap O_{x}$. On choisit des ouverts-fermés $V$ et $W$ tels que $(x, y) \in V \times W$ et $V \times W \subseteq O \cap(U \times T)$. On peut trouver $q$ tel que $k_{q}(x) \in W$ et un ouvert-fermé non vide $V^{\prime} \subseteq k_{q}^{-1}(W) \cap V$; si $z \in V^{\prime} \cap H, \overline{k[z]} \backslash l[z]$ étant dense dans $\overline{k[z]}$, on peut trouver $y(z)$ tel que $(z, y(z)) \in N \cap(V \times W)$. Donc $U \cap \Pi_{Z}[N \cap O]$ contient $V^{\prime} \cap H$, qui est non maigre. On a donc montré que pour tout ouvert-fermé non vide $U$ de $Z, U \cap \Pi_{Z}[N \cap O]$ est non maigre. Comme $\Pi_{Z}[N \cap O]$ est analytique, on en déduit que $\Pi_{Z}[N \cap O]$ est comaigre dans $Z$. D'où la condition (d) d'un système réducteur.

Soient $U$ et $V$ des ouverts-fermés tels que $N \cap(U \times V) \neq \emptyset$, et $(x, y)$ dans $N \cap(U \times V)$. Comme $x \in G(k)$ et $y \in V \cap \overline{k[x]}, k[x]$ n'a pas de point 
isolé et on peut trouver une infinité de $q$ tels que $z:=k_{q}(x) \in V$. Comme avant, on voit que $\overline{k[x]} \backslash l[x]$ est dense dans $\overline{k[x]}$, donc $z$ est limite de points $z_{n} \in V \cap \overline{k[x]} \backslash l[x]$. Donc $\left(x, z_{n}\right)$ est dans $N \cap(U \times V)$, et $(x, z)$ est dans $\operatorname{Gr}\left(k_{q}\right) \cap \bar{N} \cap(U \times V)$. Par conséquent, $\left\{q \in \omega \mid \operatorname{Gr}\left(k_{q}\right) \cap \bar{N} \cap(U \times V) \neq \emptyset\right\}$ est infini.

Posons $U_{q, p, U, V}:=U \cap k_{q, p}^{-1}(V)$. Il est clair que $\operatorname{Gr}\left(k_{q, p}\right) \cap \bar{N} \cap(U \times V)$ est inclus dans $\operatorname{Gr}\left(k_{q, p}\left\lceil U_{q, p, U, V}\right)\right.$. Réciproquement, si $(x, y)$ est élément de $\operatorname{Gr}\left(k_{q, p}\left\lceil U_{q, p, U, V}\right)\right.$, il faut voir que $(x, y) \in \bar{N}$. Or $x$ est limite d'une suite $\left(x_{n}\right) \subseteq D_{k_{q, p}} \cap H$. Comme avant, $k_{q, p}\left(x_{n}\right)$ est limite de $\left(y_{m}^{n}\right)_{m}$ incluse dans $\overline{k\left[x_{n}\right]} \backslash l\left[x_{n}\right]$, et on peut supposer que $d\left(k_{q, p}\left(x_{n}\right), y_{m}^{n}\right)<2^{-n-m}$. Alors $\left(x_{n}, y_{n}^{n}\right) \in N$ et tend vers $(x, y) \in \bar{N}$.

\section{Existence d'exemples et synthèse des résultats précédents}

Notations. Soit $\left(q_{n}\right)$ la suite des nombres premiers : $q_{0}=2, q_{1}=3$, $q_{2}=5, \ldots$ On pose

$$
J:\left\{\begin{aligned}
\omega^{<\omega} & \rightarrow \omega, \\
s & \mapsto \begin{cases}q_{0}^{s(0)+1} \ldots q_{|s|-1}^{s(|s|-1)+1} & \text { si } s \neq \emptyset, \\
0 & \text { sinon. }\end{cases}
\end{aligned}\right.
$$

On définit $A_{i}:=\{1\} \cup\left\{J(u \frown 1) \mid u \in \prod_{p<i} A_{p}\right\}$, puis $F:=\prod_{i \in \omega} A_{i}$. On pose, pour $(s, t) \in \bigcup_{n \in \omega} \omega^{n} \times \omega^{n+1}$,

$$
\begin{aligned}
D_{f_{s, t}}:=\{\alpha \in F|\forall j \leq| s \mid \alpha(J[s\lceil j \frown t\lceil(j+1)])=1 \text { et } \\
\forall p<t(j) \quad \alpha\left(J\left[s\left\lceil j^{\frown} t\left\lceil j^{\frown} p\right]\right) \neq 1\right\} .\right.
\end{aligned}
$$

On définit ensuite les fonctions

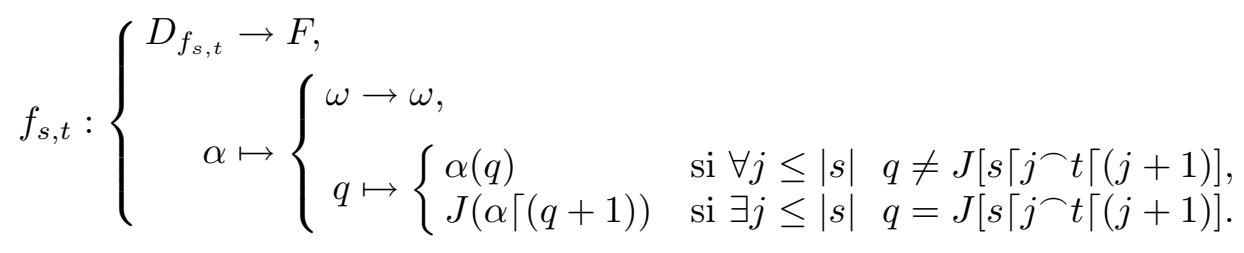

Lemme 6. Il existe une situation de départ.

Démonstration. Soient $e: \omega \rightarrow \omega^{<\omega}$ vérifiant $e^{-1}(s)<e^{-1}\left(s^{\frown} n\right)$ bijective, et aussi $\Psi: \omega^{2} \rightarrow \bigcup_{n \in \omega} \omega^{n} \times \omega^{n+1}$ et $\theta: \bigcup_{n \in \omega} \omega^{n} \times \omega^{n+1} \rightarrow \omega^{2}$ bijectives réciproques l'une de l'autre avec $\Psi_{0}(n, p)=e(n)$ et aussi $\theta_{0}\left(s, t^{\frown} m\right)=e^{-1}(s)$. Alors on pose $f_{n, p}:=f_{\Psi(n, p)}$. On va voir que $\left(F,\left(f_{n, p}\right)_{(n, p) \in \omega^{2}}\right)$ est une situation de départ.

Il est clair que $A_{i}$ est une partie finie de $\omega$, de cardinal au moins deux, de sorte que $F$ est un compact parfait non vide de $\omega^{\omega}$, donc une copie de $\mathbf{2}^{\omega}$. D'où la condition (a) d'une situation générale et la condition (b) d'une situation de départ. 
Les ensembles $D_{f_{s, t}}$ sont des ouverts-fermés de $F$, donc des compacts, et $f_{s, t}$ est clairement définie, injective et continue, donc est un homéomorphisme de $D_{f_{s, t}}$ sur son image qui vaut

$$
\begin{aligned}
\left\{\alpha \in F \mid \exists u \in \prod_{i \leq J(s \frown t)} A_{i} \forall i \leq\right. & J(s \frown t) \\
& \left.\alpha(i)=f_{s, t}\left(u \frown 1^{\omega}\right)(i) \text { et } u^{\frown 1^{\omega}} \in D_{f_{s, t}}\right\} .
\end{aligned}
$$

Cette image est ouverte-fermée dans $F$, d'où la condition (b) d'une situation générale.

Il est clair que les $D_{f_{s, t} \frown m}$, pour $m \in \omega$, sont deux à deux disjoints, donc les $D_{f_{s, t}}$, pour $t \in \omega^{|s|+1}$, aussi car $D_{f_{s, t} \frown m} \subseteq D_{f_{s\lceil(|s|-1), t}}$. Leur réunion $D_{f_{s}}$ est dense dans $F$, car si $\tilde{t} \in \prod_{i \leq k} A_{i}$, on peut trouver $t \in \omega^{|s|+1}$ telle que $\widetilde{t} 1^{\omega} \in D_{f_{s, t}}$. En effet, on peut trouver une suite finie d'entiers $\widetilde{s}$ telle que pour tout $j<|\widetilde{s}| \leq|s|+1$, on ait $\widetilde{t}(J[s\lceil j \frown \widetilde{s}\lceil(j+1)])=1$ et pour tout $p<\widetilde{s}(j), \widetilde{t}(J[s\lceil j \frown \widetilde{s}\lceil j \frown p]) \neq 1$; de plus, on peut exiger qu'elle soit de longueur maximale avec ces propriétés, c'est-à-dire que pour tout $i \in \omega$, $J[s\lceil|\widetilde{s}| \frown \widetilde{s} \frown i] \geq|\widetilde{t}|$ ou $\widetilde{t}(J[s\lceil|\widetilde{s}| \frown \widetilde{s} \frown i]) \neq 1$ ou $|\widetilde{s}|=|s|+1$. Si $|\widetilde{s}|=|s|+1$, on peut poser $t:=\widetilde{s}$. Sinon, on choisit $i$ minimal tel que $J[s\lceil|\widetilde{s}| \frown \widetilde{s} \frown i] \geq|\widetilde{t}|$ et on pose $t:=\widetilde{s} \frown i \frown 0^{|s|-|\widetilde{s}|}$. D'où la condition (c) d'une situation générale.

Si $x \in D_{s \frown n, t \frown m}$, on a $x \in D_{f_{s, t}}$ et

$$
d\left(f_{s \frown n, t \frown m}(x), f_{s, t}(x)\right) \leq 2^{-J(s \frown n \frown t \frown m)} .
$$

Donc si $x \in D_{f_{s} \frown n} \cap D_{f_{s}}$, il existe $(t, m) \in \omega^{|s|+1} \times \omega$ tel que $x \in D_{f_{s}{ }_{n, t} \frown m}$ et la suite $\left(f_{s} \frown n(x)\right)_{n}$ tend vers $f_{s}(x)$, qui n'est donc pas isolé. On a donc la condition (d) d'une situation générale, avec $G(f):=\bigcap_{n \in \omega} D_{f_{n}}$; d'où la condition (a) d'une situation de départ.

La condition (c) d'une situation de départ est clairement vérifiée. Soit donc $(x, y)$ dans $\left(\bigcap_{n \in \omega} D_{f_{n}} \times F\right) \cap \overline{\bigcup_{n \in \omega} \operatorname{Gr}\left(f_{n}\right)}$. On peut trouver $x_{k}$ et $n_{k}$ tels que $(x, y)$ soit la limite en $k$ de $\left(x_{k}, f_{n_{k}}\left(x_{k}\right)\right)$ :

$$
\forall p \in \omega \exists k(p) \in \omega \forall k \geq k(p) \quad x_{k}\left\lceil p=x\left\lceil p \text { et } f_{n_{k}}\left(x_{k}\right)\lceil p=y\lceil p .\right.\right.
$$

Montrons que la suite $\left(f_{n}\right)$ est équicontinue en $x$. Soit $q \in \omega$. Comme $x$ est dans $\bigcap_{n \in \omega} D_{f_{n}}$, les coordonnées de $x$ modifiées sont les $J(s \frown t \frown m)$, où $x \in D_{f_{s, t}{ }_{m}}, s, t \in \omega^{<\omega}$ et $|s|=|t|, m$ variant dans $\omega$. Par suite, les couples $\left(x\left\lceil q, f_{n}(x)\lceil q)\right.\right.$ sont donc en nombre fini, à $q$ fixé. Comme les $f_{n}$ sont continues, on a l'équicontinuité :

$$
\forall p \in \omega \exists q(p) \in \omega \quad z\left\lceil q(p)=x\left\lceilq ( p ) \Rightarrow \forall n \in \omega \quad f _ { n } ( z ) \left\lceil p=f_{n}(x)\lceil p .\right.\right.\right.
$$

Posons $y_{k}:=f_{n_{k}}(x)$. Alors on a $\left(x, y_{k}\right) \in \bigcup_{n \in \omega} \operatorname{Gr}\left(f_{n}\right)$ et si $p \in \omega$, soit $k \geq k(\max [p, q(p)])$. Alors $x_{k}\lceil q(p)=x\lceil q(p)$, d'où la suite d'égalités $y\left\lceil p=f_{n_{k}}\left(x_{k}\right)\left\lceil p=f_{n_{k}}(x)\left\lceil p=y_{k}\lceil p\right.\right.\right.$. Pour avoir la condition (d) d'une situation de départ, il reste à voir que $x \neq y$. Mais ceci résulte du fait que 
la première coordonnée de $x$ égale à 1 et d'ordre une puissance positive de 2 est transformée de la même façon par toutes les $f_{n_{k}}$.

Si $N_{s}^{2}$ rencontre l'un des graphes des $f_{n}$, il n'y a pas de 1 parmi les coordonnées de $s$ d'ordre une puissance positive de 2 ; par suite, $N_{s}^{2}$ rencontre le graphe de $f_{0}$. Si maintenant $x \neq y, x \frown j \mathfrak{R} y\left\ulcorner j\right.$ et $\left(N_{x} \times N_{y}\right) \cap \operatorname{Gr}\left(f_{s}\right) \neq \emptyset$, il existe $t \prec s$ telle que $\left(N_{x} \times N_{y}\right) \cap \operatorname{Gr}\left(f_{t}\right) \neq \emptyset$, avec $f_{t}$ ne changeant que les coordonnées d'ordre inférieur à $|x|$. De plus, toute suite $u$ telle que $N_{x} \times N_{y}$ rencontre $\operatorname{Gr}\left(f_{u}\right)$ vérifie $t \prec u$, d'où $e^{-1}(t) \leq e^{-1}(u)$ et $\psi(x, y)=e^{-1}(t)$. Comme $\left(N_{x \frown j} \times N_{y \frown j}\right) \cap \operatorname{Gr}\left(f_{t}\right) \neq \emptyset$, on a $\psi(x \frown j, y \frown j) \leq e^{-1}(t)=\psi(x, y)$. D'où la condition (e)(i) d'une situation de départ.

Soient $s$ et $t$ dans $\prod_{i<k} A_{i}$ et $u, v$ des $\mathfrak{T}$-chaînes sans répétition de termes telles que $u(0)=v(0)=s$ et $u(|u|-1)=v(|v|-1)=t$, avec $|u| \leq|v|$. On veut montrer que $u=v$; on peut supposer que $|u| \geq 2$ et $u(|u|-2) \neq$ $v(|v|-2)$. Posons

$$
w(i)= \begin{cases}u(i) & \text { si } i<|u| \\ v(|u|+|v|-2-i) & \text { si }|u| \leq i<|u|+|v|-1 .\end{cases}
$$

Alors $|w| \geq 3, w(0)=w(|w|-1)=s, w(i) \neq w(i+1)$ si $i<|w|-1$ et $w(i) \neq w(i+2)$ si $i<|w|-2$. Soit $c$ une $\mathfrak{T}$-chaîne de longueur minimale ayant ces propriétés, et telle que $l:=|c(0)|$ soit minimale elle aussi.

L'argument qui suit a été vu dans la preuve du théorème 2.7 de [Le3]. La suite $(c(i)(l-1))_{i<|c|}$ est non constante, et on trouve $i_{1}$ minimal tel que $c\left(i_{1}\right)(l-1) \neq c\left(i_{1}+1\right)(l-1)$; il y a alors deux cas.

Ou bien $c\left(i_{1}\right)(l-1)<c\left(i_{1}+1\right)(l-1)$, auquel cas comme on a les égalités $c\left(i_{1}\right)(l-1)=c(0)(l-1)=c(|c|-1)(l-1)$, on trouve $i_{2}>i_{1}+1$ minimal tel que $c\left(i_{1}+1\right)(l-1) \neq c\left(i_{2}\right)(l-1)$. On a $c\left(i_{1}\right)=c\left(i_{2}\right)$, par injectivité de $J$. Donc $i_{1}=0$ et $i_{2}=|c|-1$, par minimalité de $|c|$. Par minimalité encore, $|c|=3$, ce qui constitue la contradiction cherchée (on a $c\left(i_{1}+1\right)=$ $c\left(i_{2}-1\right)$ car il existe un unique couple $(s, t)$ tel que $c\left(i_{1}\right) \frown 1^{\omega} \in D_{f_{s, t}}$, avec $J(s \frown t)=l-1$; par suite, on a la suite d'égalités $c\left(i_{1}+1\right) \frown 1^{\omega}=$ $\left.f_{s, t}\left(c\left(i_{1}\right) \frown 1^{\omega}\right)=f_{s, t}\left(c\left(i_{2}\right) \frown 1^{\omega}\right)=c\left(i_{2}-1\right) \frown 1^{\omega}\right)$.

Ou bien $c\left(i_{1}\right)(l-1)>c\left(i_{1}+1\right)(l-1)$, auquel cas on trouve $i_{2}>i_{1}+1$ minimal tel que $c\left(i_{2}\right)(l-1)=\ldots=c(|c|-1)(l-1)$. On a $c\left(i_{1}+1\right)=c\left(i_{2}-1\right)$, donc $c\left(i_{1}\right)=c\left(i_{2}\right)$ comme avant. D'où $i_{1}=0$ et $i_{2}=|c|-1$, par minimalité de $|c|$. Par minimalité encore, $|c|=3$, ce qui constitue la contradiction cherchée. D'où la condition (e)(ii) d'une situation de départ.

THÉORÈme 7. Il existe un borélien $B$ de $\omega^{\omega} \times \omega^{\omega}$ tel que pour tous espaces polonais $X$ et $Y$, et pour tout borélien $A$ de $X \times Y$ dont les coupes horizontales et verticales sont dénombrables, on a l'équivalence entre les conditions suivantes : 
(a) Le borélien $A$ n'est pas $\operatorname{pot}\left(\mathbf{\Pi}_{2}^{0}\right)$.

(b) Il existe $u: \omega^{\omega} \rightarrow X$ et $v: \omega^{\omega} \rightarrow Y$, homéomorphismes sur leurs images, tels que $\bar{B} \cap(u \times v)^{-1}(A)=B$.

Démonstration. Soit $\left(F,\left(f_{n, p}\right)_{(n, p) \in \omega^{2}}\right)$ la situation de départ fournie par le lemme 6 . L'ensemble $G(f)=\bigcap_{n \in \omega} D_{f_{n}}$ est $G_{\delta}$ dense de $F$, donc polonais parfait de dimension 0 , et $G(f)$ est localement non compact car son complémentaire contient l'ensemble dense $D$ des suites différentes de 1 à partir d'un certain rang. On peut donc trouver un homéomorphisme $\phi_{0}: \omega^{\omega} \rightarrow G(f)$. On remarque que si $x \in G(f)$ et $n \in \omega$, on a $f_{n}(x) \notin D$, à cause de la condition (c) d'une situation de départ. Soit donc $\psi_{0}: \omega^{\omega} \rightarrow$ $F \backslash D$ un homéomorphisme. On pose $B:=\left(\phi_{0} \times \psi_{0}\right)^{-1}\left(\bigcup_{n \in \omega} \operatorname{Gr}\left[f_{n}\lceil G(f)]\right)\right.$.

Si $A$ est $\operatorname{pot}\left(\Pi_{2}^{0}\right)$, alors la condition (b) n'est pas vérifiée, car sinon $B$ serait $\operatorname{pot}\left(\boldsymbol{\Pi}_{2}^{0}\right)$, donc $\bigcup_{n \in \omega} \operatorname{Gr}\left(f_{n}\lceil G(f))\right.$ aussi. On pourrait donc trouver un $G_{\delta}$ dense $K$ de $F$ tel que pour tout $x$ de $G(f), f[x] \cap K$ soit $G_{\delta}$ de $K$, donc polonais. Mais $\left\{x \in G(f) \mid x \in \bigcap_{n \in \omega} f_{n}^{-1}(K)\right\}$ est $G_{\delta}$ dense de $G(f)$, donc on pourrait trouver $x$ dans $G(f)$ tel que $f[x]$ soit polonais, ce qui contredit le fait qu'il soit sans point isolé.

Si $A$ n'est pas $\operatorname{pot}\left(\boldsymbol{\Pi}_{2}^{0}\right)$, nous allons construire des applications $u$ et $v$ vérifiant la condition (b). Le lemme 2 fournit un système réducteur $\left(Z, T,\left(h_{n, p}\right)_{(n, p) \in \omega^{2}}, M\right)$ et des injections continues $u_{0}: Z \rightarrow X, v_{0}: T \rightarrow Y$ tels que $\bigcup_{(n, p) \in \omega^{2} \operatorname{Gr}\left(h_{n, p}\right) \subseteq\left(u_{0} \times v_{0}\right)^{-1}(A)}$ et $M \subseteq\left(u_{0} \times v_{0}\right)^{-1}(\check{A})$. Par le théorème 3 , on trouve une injection $\Psi: \omega^{2} \rightarrow \omega^{2}$ et des ouverts-fermés $D_{r, p}^{\prime} \subseteq D_{h_{\Psi(r, p)}}$ tels que si $l_{r, p}:=h_{\Psi(r, p)}\left\lceil D_{r, p}^{\prime},\left(Z, T,\left(l_{r, p}\right)_{(r, p) \in \omega^{2}}\right)\right.$ soit une situation générale et pour tout $x \in G(l), l[x] \subseteq\left\{h_{n, p}(x) \mid(n, p) \in \omega^{2}\right.$ et $\left.x \in D_{h_{n, p}}\right\}$, et pour tout $y \in \overline{l[x]} \backslash l[x],(x, y) \in M$. Par le théorème 4 , on trouve une situation d'arrivée $\left(Z, T,\left(k_{q, p}\right)_{(q, p) \in \omega^{2}}\right)$ telle que $G(k) \subseteq G(l)$ et pour $x$ dans $G(k)$, on ait $k[x] \subseteq l[x]$. Par le lemme 5 , on trouve un ensemble $N, G_{\delta}$ de $Z \times T$, tel que $\left(Z, T,\left(k_{q, p}\right)_{(q, p) \in \omega^{2}}, N\right)$ soit un système réducteur et $N \cap \bigcup_{r \in \omega} \operatorname{Gr}\left(l_{r}\right)=\emptyset$. Par le théorème 3 encore, on trouve une injection $\Phi: \omega^{2} \rightarrow \omega^{2}$ et des ouverts-fermés $D_{m, p} \subseteq D_{k_{\Phi(m, p)}}$ tels que si $g_{m, p}:=$ $k_{\Phi(m, p)}\left\lceil D_{m, p},\left(Z, T,\left(g_{m, p}\right)_{(m, p) \in \omega^{2}}\right)\right.$ soit une situation d'arrivée et pour $x$ dans $G(g) \subseteq G(k), g[x] \subseteq k[x]$ et pour tout $y$ de $\overline{g[x]} \backslash g[x],(x, y) \in N$. Par le théorème 1 , on trouve des injections continues $u_{1}: F \rightarrow G(g)$ et $v_{1}: F \rightarrow T$ telles que pour $(x, y)$ dans $\bigcup_{n \in \omega} \operatorname{Gr}\left(f_{n}\right)$ on ait $\left(u_{1}(x), v_{1}(y)\right) \in \bigcup_{m \in \omega} \operatorname{Gr}\left(g_{m}\right)$ et pour tout $(x, y) \in \bigcap_{n \in \omega} D_{f_{n}} \times T \cap \overline{\bigcup_{n \in \omega} \operatorname{Gr}\left(f_{n}\right)} \backslash \bigcup_{n \in \omega} \operatorname{Gr}\left(f_{n}\right), v_{1}(y)$ soit dans $\overline{g\left[u_{1}(x)\right]} \backslash g\left[u_{1}(x)\right]$.

On pose alors $u:=u_{0}\left\lceil G(g) \circ u_{1}\left\lceil G(f) \circ \phi_{0}, v:=v_{0} \circ v_{1}\left\lceil(F \backslash D) \circ \psi_{0}\right.\right.\right.$. Comme $u_{0}\left\lceil G(g) \circ u_{1}\right.$ et $v_{0} \circ v_{1}$ sont des homéomorphismes sur leurs images, $u$ et $v$ aussi. Si $(x, y) \in B,\left(\phi_{0}(x), \psi_{0}(y)\right) \in \bigcup_{n \in \omega} \operatorname{Gr}\left(f_{n}\lceil G(f))\right.$ donc $\left(u_{1}\left[\phi_{0}(x)\right], v_{1}\left[\psi_{0}(y)\right]\right) \in \bigcup_{m \in \omega} \operatorname{Gr}\left(g_{m}\right)$ et $u_{1}\left[\phi_{0}(x)\right] \in G(g) \subseteq G(k) \subseteq G(l)$. 
Par suite, on a

$$
\begin{aligned}
v_{1}\left[\psi_{0}(y)\right] & \in g\left[u_{1}\left[\phi_{0}(x)\right]\right] \subseteq k\left[u_{1}\left[\phi_{0}(x)\right]\right] \subseteq l\left[u_{1}\left[\phi_{0}(x)\right]\right] \\
& \subseteq\left\{h_{n, p}\left(u_{1}\left[\phi_{0}(x)\right]\right) \mid(n, p) \in \omega^{2} \text { et } u_{1}\left[\phi_{0}(x)\right] \in D_{h_{n, p}}\right\} .
\end{aligned}
$$

Donc $(u(x), v(y)) \in A$. Si $(x, y) \in \bar{B} \backslash B$, on a

$$
\left(\phi_{0}(x), \psi_{0}(y)\right) \in \overline{\bigcup_{n \in \omega} \operatorname{Gr}\left(f_{n}\lceil G(f))\right.} \backslash \bigcup_{n \in \omega} \operatorname{Gr}\left[f_{n}\lceil G(f)] .\right.
$$

Comme $\phi_{0}(x) \in G(f) \subseteq \bigcap_{n \in \omega} D_{f_{n}}$, on a

$$
\left(\phi_{0}(x), \psi_{0}(y)\right) \in \overline{\bigcup_{n \in \omega} \operatorname{Gr}\left(f_{n}\right)} \backslash \bigcup_{n \in \omega} \operatorname{Gr}\left(f_{n}\right) .
$$

Par conséquent, $v_{1}\left[\psi_{0}(y)\right] \in \overline{g\left[u_{1}\left[\phi_{0}(x)\right]\right]} \backslash g\left[u_{1}\left[\phi_{0}(x)\right]\right]$. Comme $u_{1}\left[\phi_{0}(x)\right]$ est dans $G(g),\left(u_{1}\left[\phi_{0}(x)\right], v_{1}\left[\psi_{0}(y)\right]\right)$ est dans $N$, et donc que $v_{1}\left[\psi_{0}(y)\right]$ appartient à $\overline{l\left[u_{1}\left[\phi_{0}(x)\right]\right]} \backslash l\left[u_{1}\left[\phi_{0}(x)\right]\right]$. Donc $\left(u_{1}\left[\phi_{0}(x)\right], v_{1}\left[\psi_{0}(y)\right]\right) \in M$ et $(u(x), v(y)) \notin A$.

En analysant cette démonstration, on obtient d'autres caractérisations des boréliens à coupes dénombrables n'étant pas $\operatorname{pot}\left(\boldsymbol{\Pi}_{2}^{0}\right)$. Le corollaire qui suit est à rapprocher du théorème 2.11 de [Le2].

Corollaire 8. Soient $X$ et $Y$ des espaces polonais, et $A$ un borélien de $X \times Y$ dont les coupes horizontales et verticales sont dénombrables. Les conditions suivantes sont équivalentes :

(a) Le borélien A n'est pas $\operatorname{pot}\left(\boldsymbol{\Pi}_{2}^{0}\right)$.

(b) Il existe une situation générale $\left(Z, T,\left(g_{m, p}\right)_{(m, p) \in \omega^{2}}\right)$ et des injections continues $i: Z \rightarrow X, j: T \rightarrow Y$ telles que pour tout $x$ de $G(g)$, on ait $\overline{g[x]} \cap(i \times j)^{-1}(A)_{x}=g[x]$.

(c) Il existe une situation d'arrivée $\left(Z, T,\left(g_{m, p}\right)_{(m, p) \in \omega^{2}}\right)$ et des injections continues $i: Z \rightarrow X, j: T \rightarrow Y$ telles que pour tout $x$ de $G(g)$, on ait $\overline{g[x]} \cap(i \times j)^{-1}(A)_{x}=g[x]$.

(d) Il existe une situation de départ $\left(F,\left(f_{n, p}\right)_{(n, p) \in \omega^{2}}\right)$ et des injections continues $u: F \rightarrow X, v: F \rightarrow Y$ telles que

$$
\overline{\bigcup_{n \in \omega} \operatorname{Gr}\left(f_{n}\right)} \cap(G(f) \times F) \cap(u \times v)^{-1}(A)=\bigcup_{n \in \omega} \operatorname{Gr}\left(f_{n}\lceil G(f)) .\right.
$$

Démonstration. Il suffit de lire la preuve du théorème 7 . Pour l'équivalence de (a) et $(\mathrm{d})$, on prend $u:=u_{0}\left\lceil G(g) \circ u_{1}\right.$ et $v:=v_{0} \circ v_{1}$. Pour l'équivalence de (a) avec (b) et (c), on prend $i:=u_{0}$ et $j:=v_{0}$, de sorte que $i$ et $j$ correspondent simplement à un changement de topologie. Ces équivalences viennent $d u$ fait que l'union $\bigcup_{m \in \omega} \operatorname{Gr}\left(g_{m}\lceil G(g))\right.$ vaut $\{(x, y) \in G(g) \times T \mid y \in \overline{g[x]}\} \cap(i \times j)^{-1}(A)$. 
Corollaire 9. Soit $\Gamma$ une classe de Wadge non stable par passage au complémentaire. Alors il existe un borélien $B_{\Gamma}$ de $\omega^{\omega} \times \omega^{\omega}$ et un fermé $F_{\Gamma}$ contenant $B_{\Gamma}$ tels que pour tous espaces polonais $X$ et $Y$, et pour tout borélien $A$ de $X \times Y$ ayant ses coupes horizontales et verticales dénombrables, on a l'équivalence entre les conditions suivantes:

(a) Le borélien $A$ n'est pas $\operatorname{pot}(\Gamma)$.

(b) Il existe des fonctions continues $u: \omega^{\omega} \rightarrow X$ et $v: \omega^{\omega} \rightarrow Y$ telles que $F_{\Gamma} \cap(u \times v)^{-1}(A)=B_{\Gamma}$.

Démonstration. Si $\Gamma$ vaut $\boldsymbol{\Pi}_{2}^{0}$, on applique le théorème 7 . Si $\Gamma$ vaut $D_{\xi}\left(\boldsymbol{\Sigma}_{1}^{0}\right)$ ou $\check{D}_{\xi}\left(\boldsymbol{\Sigma}_{1}^{0}\right)$, on applique les théorèmes 3.5 et 3.6 de [Le3] et on utilise l'existence d'une rétraction continue de $\omega^{\omega}$ sur $2^{\omega}$. Sinon, $\Gamma$ contient $\Sigma_{2}^{0}$, donc $A$ est $\operatorname{pot}(\Gamma)$. Il suffit alors de prendre $B_{\Gamma}:=\left(\omega^{\omega} \times \omega^{\omega}\right) \backslash B_{\Pi_{2}^{0}}$ et $F_{\Gamma}:=\bar{B}_{\Gamma}$, puisque $B_{\boldsymbol{\Pi}_{2}^{0}} \notin \operatorname{pot}\left(\boldsymbol{\Pi}_{2}^{0}\right)$, par le théorème 7 .

REmarque. $B_{\Pi_{2}^{0}}$ étant réunion dénombrable de graphes de fonctions continues est $\boldsymbol{\Sigma}_{2}^{0} \backslash \operatorname{pot}\left(\boldsymbol{\Pi}_{2}^{0}\right)$. Si $\Gamma \subseteq \boldsymbol{\Delta}_{2}^{0}$ et $\check{\Gamma}$ est stable par intersection avec les fermés (c'est-à-dire si $\Gamma=D_{\xi}\left(\boldsymbol{\Sigma}_{1}^{0}\right)$ avec $\xi$ impair ou $\Gamma=\check{D}_{\xi}\left(\boldsymbol{\Sigma}_{1}^{0}\right)$ avec $\xi$ pair), on a aussi $B_{\Gamma} \in \check{\Gamma} \backslash \operatorname{pot}(\Gamma)$. En effet, on applique le théorème $\mathrm{B}$ à $A \in \check{\Gamma} \backslash \operatorname{pot}(\Gamma)$ (qui existe par le théorème 3.3 de [Le1]) pour voir que $B_{\Gamma}=\bar{A}_{\xi} \backslash A_{\xi} \in \check{\Gamma}$. On en déduit que $B_{\check{\Gamma}}=A_{\xi} \in D_{\xi+1}\left(\Sigma_{1}^{0}\right)$ si $\xi$ est impair et que $B_{\check{\Gamma}} \in \check{D}_{\xi+1}\left(\boldsymbol{\Sigma}_{1}^{0}\right)$ si $\xi$ est pair. On n'a pas mieux en général (cf. [Le3] pour $\left.\xi=1: B_{\boldsymbol{\Pi}_{1}^{0}} \in D_{2}\left(\boldsymbol{\Sigma}_{1}^{0}\right) \backslash \operatorname{pot}\left(\check{D}_{2}\left(\boldsymbol{\Sigma}_{1}^{0}\right)\right)\right)$.

4. Une limite du résultat principal. On peut observer un phénomène analogue à celui décrit dans la section 2.C de [Le3], c'est-à-dire que dans le théorème 7 , en supposant seulement $A$ à coupes verticales dénombrables, on a une incompatibilité avec l'existence des injections $u$ et $v$.

DÉFInition. Soit $\left(h_{s}\right)_{s \in(\omega \backslash\{0\})<\omega}$ une suite de fonctions partielles de $\mathbf{2}^{\omega}$ dans $2^{\omega}$. On dit que $\left(h_{s}\right)_{s \in(\omega \backslash\{0\})<\omega}$ est une bonne suite si :

(a) Le domaine $D_{h_{s}}$ de $h_{s}$ est un ouvert dense de $\mathbf{2}^{\omega}$.

(b) Les fonctions $h_{s}$ sont continues et ouvertes.

(c) Si $x \in \bigcap_{s \in(\omega \backslash\{0\})<\omega} D_{h_{s}}$ et $s \in(\omega \backslash\{0\})^{<\omega}$, alors

$$
\lim _{k \rightarrow \infty} h_{s \frown k}(x)=h_{s}(x) .
$$

(d) Si $s, t \in(\omega \backslash\{0\})^{<\omega}$ et $s \neq t$, alors $\left\{x \in D_{h_{s}} \cap D_{h_{t}} \mid h_{s}(x) \neq h_{t}(x)\right\}$ est dense dans $\mathbf{2}^{\omega}$.

Exemple. Soit $J: \omega^{<\omega} \rightarrow \omega$ l'injection définie dans la section 3. On pose 


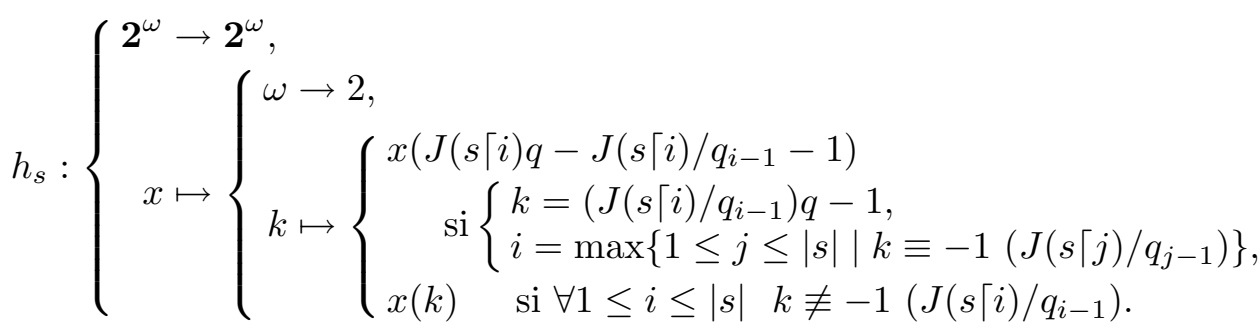

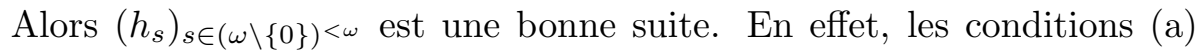
et (b) sont réalisées. Le plus petit entier $n$ tel que $h_{s \frown k}(x)(n) \neq h_{s}(x)(n)$, s'il existe, est supérieur ou égal à $J(s \frown k) / q_{|s|}-1$, qui tend vers l'infini avec $k$. D'où la condition (c). Soit $u \in 2^{<\omega}$; on cherche $x \in \mathbf{2}^{\omega}$ tel que $h_{s}(u \frown x) \neq h_{t}(u \frown x)$.

Ou bien on trouve $m<\min (|s|,|t|)$ tel que $s(m) \neq t(m)$ et $s\lceil m=t\lceil m$, avec par exemple $s(m)<t(m)$. Posons $k_{n}:=\left(J\left(t\lceil m+1) / q_{m}\right) q_{m+2}^{n}-1\right.$. On a

$h_{s}(u \frown x)\left(k_{n}\right)=u \frown x\left(J\left(s\lceil m+1) q_{m}^{t(m)-s(m)-1} q_{m+2}^{n}-J\left(s\lceil m+1) / q_{m}-1\right)\right.\right.$, $h_{t}(u \frown x)\left(k_{n}\right)=u \frown x\left(J\left(t\lceil m+1) q_{m+2}^{n}-J\left(t\lceil m+1) / q_{m}-1\right)\right.\right.$.

On choisit $n \in \omega$ tel que les numéros des coordonnées ci-dessus soient supérieurs à $|u|$. Une simplification par $q_{m}^{s(m)}$ montre que ces deux numéros sont différents. D'où l'existence de $x$.

Ou bien par exemple $s$ est un début strict de $t$. On pose alors $k_{n}:=$ $\left(J(t) / q_{|t|-1}\right) q_{|t|+1}^{n}-1$. On a

$$
\begin{aligned}
& h_{s}(u \frown x)\left(k_{n}\right) \\
& \quad=u \frown x\left(J(s) q_{|s|-1} q_{|s|}^{t(|s|)+1} \ldots q_{|t|-2}^{t(|t|-2)+1} q_{|t|-1}^{t(|t|-1)} q_{|t|+1}^{n}-J(s) / q_{|s|-1}-1\right), \\
& h_{t}(u \frown x)\left(k_{n}\right)=u \frown x\left(J(t) q_{|t|+1}^{n}-J(t) / q_{|t|-1}-1\right) .
\end{aligned}
$$

On conclut comme avant, avec simplification par $q_{|s|-1}^{s(|s|-1)}$.

Lemme 10. Soit $\left(h_{s}\right)_{s \in(\omega \backslash\{0\})<\omega}$ une bonne suite, et $G$ un $G_{\delta}$ dense de $\mathbf{2}^{\omega}$ inclus dans $\bigcap_{s \in(\omega \backslash\{0\})<\omega} D_{h_{s}} \cap \bigcap_{s \neq t}\left\{x \in D_{h_{s}} \cap D_{h_{t}} \mid h_{s}(x) \neq h_{t}(x)\right\}$. Alors $\bigcup_{s \in(\omega \backslash\{0\})<\omega} \operatorname{Gr}\left(h_{s}\lceil G)\right.$ n'est pas $\operatorname{pot}\left(G_{\delta}\right)$.

Démonstration. Elle est identique à celle du deuxième point de la preuve du théorème 7 .

LEMme 11. Soit $\left(h_{s}\right)_{s \in(\omega \backslash\{0\})<\omega}$ une bonne suite. Alors il existe une bonne suite $\left(l_{s}\right)_{s \in(\omega \backslash\{0\})^{<\omega}}$, une suite $\left(V_{s, t}\right)_{(s, t) \in \bigcup_{n \in \omega}(\omega \backslash\{0\})^{n} \times(\omega \backslash\{0\})^{n+1}}$ d'ouverts non vides de $\mathbf{2}^{\omega}$ et $\phi$ de $\bigcup_{n \in \omega}(\omega \backslash\{0\})^{n} \times(\omega \backslash\{0\})^{n+1}$ dans $(\omega \backslash\{0\})^{<\omega}$ telles que:

(a) $D_{l_{s}}=\bigcup_{t \in \omega^{|s|+1}, \text { disj. }} V_{s, t}$ et $V_{s \frown m, t \frown n} \subseteq V_{s, t}$.

(b) Pour tout $x$ de $V_{s, t}$ on a $l_{s}(x)=h_{\phi(s, t)}(x)$. 
(c) La suite $\phi(s, t)$ est un début strict de $\phi(s \frown m, t \frown n)$.

(d) On a $D_{l_{s}{ }_{k}} \subseteq D_{l_{s}} \cap \bigcap_{j<k} D_{l_{s}{ }_{j}}$ et pour tout $x$ dans $D_{l_{s}{ }_{k}}$ et tout $i \leq|s|, l_{s \frown k}(x) \neq l_{s\lceil i}(x)$.

(e) Pour tout $x$ de $D_{l_{s} \frown k}$ on a $d\left(l_{s \frown k}(x), l_{s}(x)\right)<\varepsilon(s \frown k, x)$, où $\varepsilon(s \frown k, x)$ est par définition

$$
\min \left[2^{-k}, \min _{i<|s|} \frac{1}{4} d\left(l_{s\lceil i+1}(x), l_{s\lceil i}(x)\right), \min _{j<k} \frac{1}{4} d\left(l_{s \frown j}(x), l_{s}(x)\right)\right] .
$$

Démonstration. On commence par poser

$$
V_{\emptyset, n}:=\left\{x \in D_{h_{\emptyset}} \mid x(n)=1 \text { et } \forall p<n \quad x(p)=0\right\}, \quad \phi(\emptyset, n):=\emptyset .
$$

Admettons avoir construit $l_{y}$ pour $y$ débutant $s$, et pour $y=s \frown j$ avec $j<k$. On va construire $l_{s \frown k}$. Soit $t \in \omega^{|s|+1}$ et $\left(x_{n}\right)$ une suite dense de l'ensemble suivant :

$V_{s, t} \cap D_{l_{s}} \cap \bigcap_{j<k} D_{l_{s} \frown j} \cap \bigcap_{w \in(\omega \backslash\{0\})<\omega} D_{h_{w}} \cap \bigcap_{u \neq v}\left\{x \in D_{h_{u}} \cap D_{h_{v}} \mid h_{u}(x) \neq h_{v}(x)\right\}$.

Par hypothèse de récurrence, $\varepsilon(s \frown k, \cdot): D_{l_{s}} \cap \bigcap_{j<k} D_{l_{s} \frown_{j}} \rightarrow \mathbb{R}_{+}^{*}$ est définie et continue; on peut donc trouver un voisinage ouvert $V$ de $x_{0}$ tel que $\varepsilon(s \frown k, x)>\frac{1}{2} \varepsilon\left(s \frown k, x_{0}\right)$ pour tout $x$ de $V$. La condition (c) d'une bonne suite fournit $r_{0} \geq k$ tel que $d\left(h_{\phi(s, t) \frown r_{0}}\left(x_{0}\right), h_{\phi(s, t)}\left(x_{0}\right)\right)<\frac{1}{2} \varepsilon\left(s \frown k, x_{0}\right)$. Par continuité de $h_{\phi(s, t)}-r_{0}$ et $h_{\phi(s, t)}$, on trouve un voisinage ouvert $W$ de $x_{0}$ tel que pour tout $x$ de $W$, on ait $d\left(h_{\phi(s, t) \frown r_{0}}(x), h_{\phi(s, t)}(x)\right)<\frac{1}{2} \varepsilon\left(s \frown k, x_{0}\right)$ et $x$ soit dans l'ensemble suivant :

$V \cap V_{s, t} \cap D_{l_{s}} \cap \bigcap_{j<k} D_{l_{s} \frown j} \cap\left\{z \in D_{h_{\phi(s, t)}{ }_{r_{0}}}|\forall i \leq| s \mid h_{\phi(s, t) \frown r_{0}}(z) \neq l_{s\lceil i}(z)\right\}$.

On pose $\phi(s \frown k, t \frown 0):=\phi(s, t) \frown r_{0}$. On choisit $V_{s \frown k, t \frown 0}$ contenant $x_{0}$ dans $W$ tel que $V_{s, t} \backslash \bar{V}_{s \frown k, t \frown 0} \neq \emptyset$. Puis on recommence ceci en remplaçant $x_{0}$ par $x_{n}$, avec $n$ minimal tel que $x_{n} \notin \bar{V}_{s \frown k, t \frown 0}$. Le choix de $V_{s \frown k, t \frown 1}$ se fait

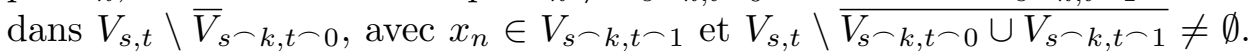
En itérant cette construction, on construit $l_{s \frown k}, V_{s \frown k, t \frown m}$ et $\phi(s \frown k, t \frown m)$ vérifiant les propriétés demandées. En effet, on a $V_{s, t} \subseteq \overline{\bigcup_{m \in \omega} V_{s \frown k, t \frown m}}$, donc $\bigcup_{t \in \omega^{|s|+1}} V_{s, t} \subseteq \overline{\bigcup_{t \in \omega^{|s|+1}, m \in \omega} V_{s \frown k, t \frown m}}=\bar{D}_{l_{s} \frown k}$. La seule chose restant à vérifier est la condition (d) d'une bonne suite. Elle résultera immédiatement du lemme qui suit.

LEMme 12. Soit $\left(l_{s}\right)_{s \in(\omega \backslash\{0\})<\omega}$ une suite de fonctions partielles de $\mathbf{2}^{\omega}$ dans $\mathbf{2}^{\omega}$ vérifiant les conditions $(\mathrm{d})$ et (e) du lemme 11. Soient $s, t$ dans $(\omega \backslash\{0\})^{<\omega}$ et $i<\min (|s|,|t|)$ tel que $s(i)<t(i)$ et $s\lceil i=t\lceil i$. Alors pour tout $x$ de $D_{l_{s}} \cap D_{l_{t}}$ on a

$$
d\left(l_{s}(x), l_{t}(x)\right) \geq \frac{1}{3} d\left(l_{s\lceil i+1}(x), l_{s\lceil i}(x)\right) .
$$


Démonstration. On a

$$
\begin{aligned}
d\left(l_{s\lceil i+1}(x), l_{s}(x)\right) & \leq d\left(l_{s\lceil i+1}(x), l_{s\lceil i+2}(x)\right)+\ldots+d\left(l_{s\lceil|s|-1}(x), l_{s}(x)\right) \\
& \leq d\left(l_{s\lceil i+1}(x), l_{s\lceil i+2}(x)\right)\left(1+\frac{1}{4}+\ldots+\frac{1}{4|s|-i-2}\right) \\
& \leq \frac{4}{3} d\left(l_{s\lceil i+1}(x), l_{s\lceil i+2}(x)\right) \quad(\text { si }|s| \geq i+2) \\
& \left.\leq \frac{1}{3} d\left(l_{s\lceil i+1}(x), l_{s\lceil i}(x)\right) \quad \text { (même si }|s|=i+1\right) .
\end{aligned}
$$

De même, $d\left(l_{t\lceil i+1}(x), l_{t}(x)\right) \leq \frac{1}{3} d\left(l_{t\lceil i+1}(x), l_{s\lceil i}(x)\right)$, car $s\lceil i=t\lceil i$. Par ailleurs on a

D'où

$$
\begin{aligned}
d\left(l_{t\lceil i+1}(x), l_{s\lceil i}(x)\right) & \leq \frac{1}{4} d\left(l_{s\lceil i \frown t(i)-1}(x), l_{s\lceil i}(x)\right) \leq \ldots \\
& \leq \frac{1}{4^{t(i)-s(i)}} d\left(l_{s\lceil i+1}(x), l_{s\lceil i}(x)\right) \\
& \leq \frac{1}{4} d\left(l_{s\lceil i+1}(x), l_{s\lceil i}(x)\right) .
\end{aligned}
$$

$$
\begin{aligned}
d\left(l_{s\lceil i+1}(x), l_{t\lceil i+1}(x)\right) & \geq d\left(l_{s\lceil i+1}(x), l_{s\lceil i}(x)\right)-d\left(l_{t\lceil i+1}(x), l_{s\lceil i}(x)\right) \\
& \geq \frac{3}{4} d\left(l_{s\lceil i+1}(x), l_{s\lceil i}(x)\right) .
\end{aligned}
$$

On a aussi $d\left(l_{t\lceil i+1}(x), l_{t}(x)\right) \leq \frac{1}{12} d\left(l_{s\lceil i+1}(x), l_{s\lceil i}(x)\right)$. D'où

$$
\begin{aligned}
d\left(l_{s}(x), l_{t}(x)\right) \geq & d\left(l_{s\lceil i+1}(x), l_{t\lceil i+1}(x)\right) \\
& -d\left(l_{s\lceil i+1}(x), l_{s}(x)\right)-d\left(l_{t\lceil i+1}(x), l_{t}(x)\right) \\
\geq & d\left(l_{s\lceil i+1}(x), l_{s\lceil i}(x)\right)\left(\frac{3}{4}-\frac{1}{3}-\frac{1}{12}\right) \\
\geq & \frac{1}{3} d\left(l_{s\lceil i+1}(x), l_{s\lceil i}(x)\right) .
\end{aligned}
$$

Remarque. On a en fait montré que pour tout $x$ de $D_{l_{s}} \cap D_{l_{t}}$, on a $l_{s}(x) \neq l_{t}(x)$ si $s \neq t$.

LEMME 13. Soit $\left(l_{s}\right)_{s \in(\omega \backslash\{0\})<\omega}$ une suite de fonctions partielles de $\mathbf{2}^{\omega}$ dans $\mathbf{2}^{\omega}$ vérifiant les conditions du lemme 11. Soient $s \in(\omega \backslash\{0\})^{<\omega} \backslash\{\emptyset\}$, $u \in 2^{<\omega}$ et $\alpha \in N_{u} \cap \bigcap_{w \in(\omega \backslash\{0\})<\omega} D_{l_{w}}$. Alors il existe $\varepsilon(s, u, \alpha)>0$ et $v(s, u, \alpha) \in 2^{<\omega}$ tels que $u \prec v(s, u, \alpha) \prec \alpha$ et pour tout $t \in(\omega \backslash\{0\})^{<\omega}$, pour tout $x \in N_{v(s, u, \alpha)} \cap \bigcap_{w \in(\omega \backslash\{0\})<\omega} D_{l_{w}}$, on ait l'implication

$$
d\left(l_{s}(x), l_{t}(x)\right)<\varepsilon(s, u, \alpha) \Rightarrow s \text { et } t \text { sont compatibles. }
$$

Démonstration. Posons, pour $y \in(\omega \backslash\{0\})^{|s|}$,

$$
\eta(y, x):=d\left(l_{y}(x), l_{s\lceil|s|-1}(x)\right) .
$$


Alors la fonction $\eta(y, \cdot): D_{l_{y}} \cap D_{l_{s\lceil|s|-1}} \rightarrow \mathbb{R}_{+}^{*}$ est définie et continue, donc on peut trouver $v(s, u, \alpha) \in 2^{<\omega}$ telle que $u \prec v(s, u, \alpha) \prec \alpha$ et pour tout $x$ de $N_{v(s, u, \alpha)} \cap \bigcap_{w \in(\omega \backslash\{0\})<\omega} D_{l_{w}}$, on ait $\eta(y, x)>\frac{3}{4} \eta(y, \alpha)$ et $\varepsilon(y, x)>\frac{3}{4} \varepsilon(y, \alpha)$, ceci pour $y \in(\omega \backslash\{0\})^{|s|}$ vérifiant

$$
y=s \text { ou }(\exists ! i<|s| y(i) \neq s(i) \text { et } \exists i<|s| \quad y(i)<s(i)) .
$$

Notons $Y$ l'ensemble de ces suites $y$. On pose

$$
\varepsilon(s, u, \alpha):=\min _{y \in Y} \min \left(\frac{1}{4} \eta(y, \alpha), \varepsilon(y, \alpha)\right) .
$$

Soient $t \in(\omega \backslash\{0\})^{<\omega}$ et $x \in N_{v(s, u, \alpha)} \cap \bigcap_{w \in(\omega \backslash\{0\})^{<\omega}} D_{l_{w}}$ tels que l'on a $d\left(l_{s}(x), l_{t}(x)\right)<\varepsilon(s, u, \alpha)$. On raisonne par l'absurde, ce qui fournit $i<\min (|s|,|t|)$ minimal tel que $s(i) \neq t(i)$.

Ou bien $s(i)<t(i)$; par le lemme 12 , on a

$$
d\left(l_{s}(x), l_{t}(x)\right) \geq \frac{1}{3} d\left(l_{s\lceil i+1}(x), l_{s\lceil i}(x)\right) .
$$

Si $|s| \geq i+2$, on a donc $d\left(l_{s}(x), l_{t}(x)\right) \geq \frac{4}{3} \varepsilon(s, x)>\varepsilon(s, \alpha) \geq \varepsilon(s, u, \alpha)$. Si $|s|=i+1$, on a $d\left(l_{s}(x), l_{t}(x)\right) \geq \frac{1}{3} \eta(s, x)>\frac{1}{4} \eta(s, \alpha) \geq \varepsilon(s, u, \alpha)$. Dans tous les cas, on a une contradiction.

Ou bien $t(i)<s(i)$; par le lemme 12 , on a

$$
d\left(l_{s}(x), l_{t}(x)\right) \geq \frac{1}{3} d\left(l_{t\lceil i+1}(x), l_{t\lceil i}(x)\right) .
$$

Si $|s| \geq i+2$, on a

$$
\begin{aligned}
d\left(l_{s}(x), l_{t}(x)\right) & \geq \frac{4}{3} \varepsilon(s\lceil i \frown t(i) \frown s(i+1), x)>\varepsilon(s\lceil i \frown t(i) \frown s(i+1), \alpha) \\
& \geq \varepsilon(s, u, \alpha) .
\end{aligned}
$$

Si $|s|=i+1$, on a

$$
d\left(l_{s}(x), l_{t}(x)\right) \geq \frac{1}{3} \eta\left(s\lceil i \frown t(i), x)>\frac{1}{4} \eta(s\lceil i \frown t(i), \alpha) \geq \varepsilon(s, u, \alpha) .\right.
$$

Là encore, on a une contradiction dans les deux cas.

LEMme 14. Soit $\left(l_{s}\right)_{s \in(\omega \backslash\{0\})<\omega}$ la bonne suite fournie par le lemme 11 , associée à la suite $\left(h_{s}\right)_{s \in(\omega \backslash\{0\})<\omega}$ de l'exemple. Supposons que $s_{i}$ soient dans $(\omega \backslash\{0\})^{<\omega}$, pour $i \in 4$, et que $t_{i} \in(\omega \backslash\{0\})^{\left|s_{i}\right|+1}$ vérifient les conditions suivantes:
(a) $\emptyset \neq s_{i} \prec \neq s_{i+1}$.
(b) $\emptyset \neq D_{s_{i}} \subseteq V_{s_{i}, t_{i}}$.
(c) $D_{s_{i+1}} \subseteq D_{s_{i}}$.
(d) $\operatorname{Im}\left(l_{i+1}^{\prime}\right) \subseteq \operatorname{Im}\left(l_{i}^{\prime}\right)$, ò̀ $l_{i}^{\prime}:=l_{s_{i}}\left\lceil D_{s_{i}}\right.$.

Alors $l_{0}^{\prime}$ ou $l_{1}^{\prime}$ 'est pas injective.

Démonstration. Raisonnons par l'absurde. Comme $D_{s_{i}} \subseteq V_{s_{i}, t_{i}}$, on a, pour tout $x$ de $D_{s_{i}}, l_{i}^{\prime}(x)=l_{s_{i}}(x)=h_{\phi\left(s_{i}, t_{i}\right)}(x)$. Comme $D_{s_{i+1}} \subseteq D_{s_{i}}$, on a $D_{s_{i+1}} \subseteq V_{s_{i+1}, t_{i+1}} \cap V_{s_{i}, t_{i}}$, donc cette intersection est non vide. Comme 
$V_{s \frown m, t \frown n} \subseteq V_{s, t}$ et $s_{i} \prec \neq s_{i+1}$, on a $t_{i} \prec \neq t_{i+1}$, par disjonction de $\left(V_{s, t}\right)_{t \in \omega^{|s|+1}}$. Donc $\phi\left(s_{i}, t_{i}\right) \prec \neq \phi\left(s_{i+1}, t_{i+1}\right)$. Posons donc

$$
u_{i}:=\phi\left(s_{i}, t_{i}\right), \quad D_{u_{i}}:=D_{s_{i}}, \quad h_{i}^{\prime}:=h_{u_{i}}\left\lceil D_{u_{i}} .\right.
$$

On a $\emptyset \neq u_{i} \prec \neq u_{i+1}, \emptyset \neq D_{u_{i}}$ et $D_{u_{i+1}} \subseteq D_{u_{i}}$. Les fonctions $h_{i}^{\prime}$ et $l_{i}^{\prime}$ sont égales, donc $\operatorname{Im}\left(h_{i+1}^{\prime}\right) \subseteq \operatorname{Im}\left(h_{i}^{\prime}\right)$ et $h_{0}^{\prime}, h_{1}^{\prime}$ sont injectives. De plus, on a, puisque $s_{2} \neq s_{3}, h_{2}^{\prime}(x)=l_{s_{2}}(x) \neq l_{s_{3}}(x)=h_{3}^{\prime}(x)$ pour tout $x$ de $D_{u_{3}}$. Pour avoir la contradiction cherchée, il suffit donc de voir que pour tout $x$ de $D_{u_{3}}$, on a

$$
h_{0}^{\prime}\left(h_{1}^{\prime-1}\left[h_{2}^{\prime}(x)\right]\right)=h_{0}^{\prime}\left(h_{1}^{\prime-1}\left[h_{3}^{\prime}(x)\right]\right) .
$$

Posons $H_{j}:=h_{0}^{\prime} \circ h_{1}^{\prime-1} \circ h_{j}^{\prime}$, pour $j=2,3$, et soit $k \in \omega$.

1. Pour tout $1 \leq i \leq\left|u_{0}\right|, k \not \equiv-1\left(J\left(u_{0}\lceil i) / q_{i-1}\right)\right.$.

On a alors $H_{j}(x)(k)=h_{1}^{\prime-1}\left[h_{j}^{\prime}(x)\right](k)=h_{1}^{\prime}\left(h_{1}^{\prime-1}\left[h_{j}^{\prime}(x)\right]\right)(k)$ car pour tout $1 \leq i \leq\left|u_{1}\right|, k \not \equiv-1\left(J\left(u_{1}\lceil i) / q_{i-1}\right)\right.$. D'où $H_{j}(x)(k)=h_{j}^{\prime}(x)(k)=x(k)$ car pour tout $1 \leq i \leq\left|u_{j}\right|, k \not \equiv-1\left(J\left(u_{j}\lceil i) / q_{i-1}\right)\right.$.

2. Il existe $1 \leq i \leq\left|u_{0}\right|$ maximal tel que $k \equiv-1\left(J\left(u_{0}\lceil i) / q_{i-1}\right)\right.$, et $q$ tel que $k=\left(J\left(u_{0}\lceil i) / q_{i-1}\right) q-1\right.$.

2.1. L'entier $i$ est maximal sous $\left|u_{1}\right|$ tel que $k \equiv-1\left(J\left(u_{1}\lceil i) / q_{i-1}\right)\right.$.

On a alors

$$
\begin{aligned}
H_{j}(x)(k) & =h_{1}^{\prime-1}\left[h_{j}^{\prime}(x)\right]\left(J \left(u_{0}\lceil i) q-J\left(u_{0}\lceil i) / q_{i-1}-1\right)\right.\right. \\
& =h_{1}^{\prime}\left(h_{1}^{\prime-1}\left[h_{j}^{\prime}(x)\right]\right)(k)=h_{j}^{\prime}(x)(k) \\
& =x\left(J \left(u_{0}\lceil i) q-J\left(u_{0}\lceil i) / q_{i-1}-1\right)\right.\right.
\end{aligned}
$$

2.2. L'entier $k$ est de la forme

$$
\frac{J\left(u_{1}\left\lceil\left|u_{0}\right|+1\right)\right.}{q_{\left|u_{0}\right|}} q^{\prime}-1
$$

(d'où $q=q_{\left|u_{0}\right|-1} q_{\left|u_{0}\right|}^{u_{1}\left(\left|u_{0}\right|\right)} q^{\prime}$ ).

On a alors $H_{j}(x)(k)=h_{1}^{\prime-1}\left[h_{j}^{\prime}(x)\right]\left(J\left(u_{0}\right) q-J\left(u_{0}\right) / q_{\left|u_{0}\right|-1}-1\right)$. Si on a $1 \leq l<\left|u_{1}\right|$ et $q^{\prime \prime}$ n'est pas multiple de $q_{l-1} q_{l}^{u_{1}(l)}, J\left(u_{0}\right) q-J\left(u_{0}\right) / q_{\left|u_{0}\right|-1}-1$ est différent de $J\left(u_{1}\lceil l) q^{\prime \prime}-J\left(u_{1}\lceil l) / q_{l-1}-1\right.\right.$ (on simplifie par $q_{\left|u_{0}\right|-1}^{u_{0}\left(\left|u_{0}\right|-1\right)}$ si $l>\left|u_{0}\right|$, et par $q_{l-1}^{u_{0}(l-1)}$ si $l<\left|u_{0}\right|$; si $l=\left|u_{0}\right|$, on obtient $q^{\prime \prime}=q=$ $q_{\left|u_{0}\right|-1} q_{\left|u_{0}\right|}^{u_{1}\left(\left|u_{0}\right|\right)} q^{\prime}$, ce qui est exclus). De même, pour tout $q^{\prime \prime}$ on a

$$
J\left(u_{0}\right) q-J\left(u_{0}\right) / q_{\left|u_{0}\right|-1}-1 \neq J\left(u_{1}\right) q^{\prime \prime}-J\left(u_{1}\right) / q_{\left|u_{1}\right|-1}-1 .
$$

Par ailleurs,

$$
J\left(u_{0}\right) q-J\left(u_{0}\right) / q_{\left|u_{0}\right|-1}-1=J\left(u_{1}\left\lceil\left|u_{0}\right|\right) / q_{\left|u_{0}\right|-1}\left(q_{\left|u_{0}\right|-1} q-1\right)-1 ;\right.
$$


comme $h_{1}^{\prime}$ est injective, la coordonnée numéro $J\left(u_{0}\right) q-J\left(u_{0}\right) / q_{\left|u_{0}\right|-1}-1$ est constante sur $D_{s_{1}}$, d'où $H_{2}(x)=H_{3}(x)$.

ThÉORÈme 15. Le théorème 7 devient faux si on suppose seulement $A$ à coupes verticales dénombrables.

Démonstration. On raisonne par l'absurde, ce qui fournit un borélien $B_{1}$. Avec $A=B$, on voit que $B_{1}$ a ses coupes horizontales et verticales dénombrables. Avec $A=B_{1}$, on voit que $B_{1} \notin \operatorname{pot}\left(G_{\delta}\right)$. Par le corollaire 8 , on obtient une situation d'arrivée $\left(Z, T,\left(g_{m, p}\right)_{(m, p) \in \omega^{2}}\right)$ et des injections continues $i: Z \rightarrow \omega^{\omega}, j: T \rightarrow \omega^{\omega}$ telles que pour tout $x$ dans $G(g)$ on ait $\overline{g[x]} \cap(i \times j)^{-1}\left(B_{1}\right)_{x}=g[x]$. Par le théorème 1 , on trouve des injections continues $\widetilde{u}: F \rightarrow G(g)$ et $\widetilde{v}: F \rightarrow T$ telles que pour tout $(x, y) \in \bigcup_{n \in \omega} \operatorname{Gr}\left(f_{n}\right)$, on ait $\widetilde{v}(y) \in g[\widetilde{u}(x)]$, et pour $(x, y)$ appartenant à $\left(\bigcap_{n \in \omega} D_{f_{n}} \times f_{n} \quad \times\right.$ $\overline{\bigcup_{n \in \omega} \operatorname{Gr}\left(f_{n}\right)} \backslash \bigcup_{n \in \omega} \operatorname{Gr}\left(f_{n}\right)$, on ait $\widetilde{v}(y) \in \overline{g[\widetilde{u}(x)]} \backslash g[\widetilde{u}(x)]$. Soit $\left(l_{s}\right)_{s \in(\omega \backslash\{0\})<\omega}$ la bonne suite fournie par le lemme 11 appliqué à la suite $\left(h_{s}\right)_{s \in(\omega \backslash\{0\})<\omega}$ de l'exemple. En appliquant le lemme 10 à la bonne suite $\left(l_{s}\right)_{s \in(\omega \backslash\{0\})<\omega}$ et à $G:=\bigcap_{s \in(\omega \backslash\{0\})<\omega} D_{l_{s}}$, on voit que

$$
A:=\bigcup_{s \in(\omega \backslash\{0\})<\omega} \operatorname{Gr}\left(l_{s}\lceil G) \notin \operatorname{pot}\left(G_{\delta}\right) .\right.
$$

Par suite, on peut trouver des injections continues $u: \omega^{\omega} \rightarrow G$ et $v: \omega^{\omega} \rightarrow \mathbf{2}^{\omega}$ telles que $\bar{B}_{1} \cap(u \times v)^{-1}(A)=B_{1}$. Posons $U:=u \circ i \circ \widetilde{u}$ et $V:=v \circ j \circ \widetilde{v} ; U$ et $V$ sont des homéomorphismes sur leurs images (incluses respectivement dans $G$ et $\left.\mathbf{2}^{\omega}\right)$ et si $(x, y)$ est dans $\bigcup_{n \in \omega} \operatorname{Gr}\left(f_{n}\right)$, on a $(U(x), V(y)) \in A$. De plus,

$$
\overline{\bigcup_{n \in \omega} \operatorname{Gr}\left(f_{n}\lceil G(f))\right.} \cap\left(U\lceil G(f) \times V)^{-1}(A)=\bigcup_{n \in \omega} \operatorname{Gr}\left(f_{n}\lceil G(f)) .\right.\right.
$$

On va montrer un résultat intermédiaire. Soient $(s, t) \in \bigcup_{n \in \omega} \omega^{n} \times \omega^{n+1}$, $X$ un ouvert non vide de $D_{f_{s, t}}$, et $\widetilde{s}, \widetilde{t} \in(\omega \backslash\{0\})^{<\omega} \backslash\{\emptyset\}$ tels que pour tout $x \in X, U(x) \in V_{\widetilde{s}, \widetilde{t}}$ et $\left(U(x), V\left(f_{s, t}(x)\right)\right) \in \operatorname{Gr}\left(l_{\widetilde{s}}\right)$. Alors on peut trouver un ouvert non vide $Y$ de $X$, des entiers $m$ et $n$, et $\widetilde{s}^{\prime}, \widetilde{t}^{\prime} \in(\omega \backslash\{0\})^{<\omega}$ tels que :

(a) La suite $\widetilde{s}$ est un début strict de $\widetilde{s}^{\prime}$.

(b) L'ouvert $Y$ est inclus dans $D_{f_{s} \frown m, t \frown n}$.

(c) Pour tout $x$ de $Y, U(x) \in V_{\widetilde{s}^{\prime}, \widetilde{t}^{\prime}}$ et $\left(U(x), V\left(f_{s \frown m, t \frown n}(x)\right)\right) \in \operatorname{Gr}\left(l_{\widetilde{s}^{\prime}}\right)$.

(d) L'ensemble $l_{\widetilde{s}^{\prime}}[U[Y]]$ est inclus dans $l_{\widetilde{s}}[U[X]]$.

Soit $O$ (respectivement $P$ ) un ouvert de $D_{l_{\tilde{s}}}\left(\right.$ respectivement $\left.\mathbf{2}^{\omega}\right)$ tel que $U[X]=U[F] \cap O$ (respectivement $\left.V\left[f_{s, t}[X]\right]=V[F] \cap P\right)$. Fixons $\alpha$ dans $U[X \cap G(f)], u^{\prime} \in 2^{<\omega}$ tel que $\alpha \in N_{u^{\prime}} \subseteq O$, et, en utilisant le lemme 13 , 


$$
\begin{aligned}
W:=\left\{(x, y) \in N_{v\left(\widetilde{s}, u^{\prime}, \alpha\right)} \times P|\forall i<| \widetilde{s} \mid \quad y \neq l_{\widetilde{s}\lceil i}(x)\right. \text { et } \\
\left.\quad d\left(y, l_{\widetilde{s}}(x)\right)<\varepsilon\left(\widetilde{s}, u^{\prime}, \alpha\right)\right\} .
\end{aligned}
$$

Alors $W$ est ouvert de $\mathbf{2}^{\omega} \times \mathbf{2}^{\omega}$. Comme $U^{-1}(\alpha) \in G(f), f_{s \frown m}\left(U^{-1}(\alpha)\right)$ tend vers $f_{s}\left(U^{-1}(\alpha)\right)$ quand $m$ tend vers l'infini. Comme $U^{-1}(\alpha)$ est dans $X \cap U^{-1}\left(N_{v\left(\widetilde{s}, u^{\prime}, \alpha\right)}\right)$, on peut trouver un $m$ tel que $\left(U^{-1}(\alpha), f_{s \frown m}\left(U^{-1}(\alpha)\right)\right)$ soit dans $(U \times V)^{-1}(W)$. On peut trouver $t^{\prime} \in \omega^{|t|}$ et $n \in \omega$ tels que $f_{s \frown m}\left(U^{-1}(\alpha)\right)=f_{s \frown m, t^{\prime} \frown n}\left(U^{-1}(\alpha)\right)$. On a $U^{-1}(\alpha) \in D_{f_{s \frown m, t^{\prime} \frown n}} \subset D_{f_{s, t^{\prime}}}$, donc $t^{\prime}=t$ puisque $U^{-1}(\alpha) \in D_{f_{s, t}}$.

Posons

$Q:=\left\{x \in X \cap U^{-1}\left(N_{v\left(\widetilde{s}, u^{\prime}, \alpha\right)}\right) \cap D_{f_{s}{ }_{m, t}{ }_{n}} \mid\left(U(x), V\left(f_{s \frown m, t \frown n}(x)\right)\right) \in W\right\}$.

Alors $Q \subseteq \bigcup_{w \in(\omega \backslash\{0\})<\omega}\left\{x \in Q \mid\left(U(x), V\left(f_{s \frown m, t \frown n}(x)\right)\right) \in \operatorname{Gr}\left(l_{w}\right)\right\}$. Par le théorème de Baire, on peut donc trouver un ouvert non vide $Y$ de $Q$ et $\widetilde{s}^{\prime} \in(\omega \backslash\{0\})^{<\omega}$ tels que pour tout $x$ de $Y,\left(U(x), V\left(f_{s \frown m, t}{ }_{n}(x)\right)\right)$ soit dans $\operatorname{Gr}\left(l_{\widetilde{s}^{\prime}}\right)$. Par le théorème de Baire encore, on peut supposer qu'il existe $\widetilde{t^{\prime}} \in(\omega \backslash\{0\})^{\left|\widetilde{s}^{\prime}\right|+1}$ telle que pour tout $x$ de $Y, U(x) \in V_{\widetilde{s}^{\prime}, \widetilde{t}^{\prime}}$.

Les conditions (b) et (c) sont clairement vérifiées. Si $x$ est dans $Y$, on a $l_{\widetilde{s}^{\prime}}(U(x))=V\left(f_{s \frown m, t \frown n}(x)\right)$; comme $x$ est dans $Q,\left(U(x), V\left(f_{s \frown m, t \frown n}(x)\right)\right)$ est dans $W$, donc $V\left(f_{s \frown m, t \frown n}(x)\right) \in V\left[f_{s, t}[X]\right]$. Donc on trouve $y \in X$ tel que $V\left(f_{s \frown m, t \frown n}(x)\right)=l_{\widetilde{s}}(U(y))$. D'où la condition $(\mathrm{d})$. De plus, on a $V\left(f_{s \frown m, t \frown n}(x)\right) \neq l_{\widetilde{s}\lceil i}(U(x))$ si $i<|\widetilde{s}|$, et on a également

$$
d\left(l_{\widetilde{s}}(U(x)), l_{\widetilde{s}^{\prime}}(U(x))\right)<\varepsilon\left(\widetilde{s}, u^{\prime}, \alpha\right) .
$$

Donc $\widetilde{s}$ est un début de $\widetilde{s}^{\prime}$ puisque $U(x) \in N_{v\left(\widetilde{s}, u^{\prime}, \alpha\right)} \cap G$. Si $\widetilde{s}=\widetilde{s}^{\prime}$, on a successivement $V\left(f_{s \frown m, t \frown n}(x)\right)=l_{\widetilde{s}^{\prime}}(U(x))=l_{\widetilde{s}}(U(x))=V\left(f_{s, t}(x)\right)$, d'où l'égalité entre $f_{s \frown m, t \frown n}(x)$ et $f_{s, t}(x)$, qui est absurde.

Revenons à la preuve du théorème. On peut trouver $(c, d)$ dans $\bigcup_{n \in \omega} \omega^{n} \times \omega^{n+1}$ et un ouvert non vide $R$ de $D_{f_{c, d}}$ tels que pour tout $x$ de $R$ on ait $\left(U(x), V\left(f_{c, d}(x)\right)\right) \notin \operatorname{Gr}\left(l_{\emptyset}\right)$. Sinon l'ensemble $H$ défini par $\bigcap_{n \in \omega}\left\{x \in G(f) \mid\left(U(x), V\left(f_{n}(x)\right)\right) \in \operatorname{Gr}\left(l_{\emptyset}\right)\right\}$ serait $G_{\delta}$ dense de $F$ et on aurait $\bigcup_{n \in \omega} \operatorname{Gr}\left(f_{n}\lceil H)=\overline{\bigcup_{n \in \omega} \operatorname{Gr}\left(f_{n}\lceil H)\right.} \cap\left(U\lceil H \times V)^{-1}\left(\operatorname{Gr}\left(l_{\emptyset}\lceil G)\right)\right.\right.\right.$, donc $\bigcup_{n \in \omega} \operatorname{Gr}\left(f_{n}\lceil H)\right.$ serait $\operatorname{pot}\left(\boldsymbol{\Pi}_{1}^{0}\right)$ non $\operatorname{pot}\left(\boldsymbol{\Pi}_{2}^{0}\right)$, ce qui est absurde.

On a l'inclusion

$$
R \subseteq \bigcup_{w \in(\omega \backslash\{0\})<\omega}\left\{x \in R \mid\left(U(x), V\left(f_{c, d}(x)\right)\right) \in \operatorname{Gr}\left(l_{w}\right)\right\} .
$$

Par le théorème de Baire comme ci-dessus, on trouve un ouvert non vide $X$ de $R$ et $\widetilde{s}, \widetilde{t}$ dans $(\omega \backslash\{0\})<\omega \backslash\{\emptyset\}$ tels que pour tout $x$ de $X$, on ait $U(x) \in V_{\widetilde{s}, \widetilde{t}}$ et $\left(U(x), V\left(f_{c, d}(x)\right)\right) \in \operatorname{Gr}\left(l_{\widetilde{s}}\right)$. On applique le point précédent à $c$, $d$, et $X$, ce qui fournit $Y_{0}, m_{0}, n_{0}$, et $s_{0}, t_{0}$. On applique ensuite le point précédent à $c^{\frown} m_{0}, d \frown n_{0}$ et $Y_{0}$, ce qui fournit $Y_{1}, m_{1}, n_{1}$, et $s_{1}, t_{1}$. On applique ensuite le point précédent à $c \frown m_{0}^{\frown} m_{1}, d \frown n_{0} n_{1}$ et $Y_{1}$, ce qui fournit $Y_{2}, m_{2}, n_{2}$, et 
$s_{2}, t_{2}$. On applique enfin le point précédent à $c \frown m_{0} m_{1} m_{2}, d \frown n_{0} n_{1} n_{2}$ et $Y_{2}$, ce qui fournit $Y_{3}, m_{3}, n_{3}$, et $s_{3}, t_{3}$. On a $\widetilde{s} \prec \neq s_{0}$ et $s_{i} \prec \neq s_{i+1}$ si $i \in 2$, donc les $s_{i}$ sont non vides. Posons $D_{s_{i}}:=U\left[Y_{i}\right]$. Avec les notations du lemme 14, on a $\operatorname{Im}\left(l_{i+1}^{\prime}\right)=l_{s_{i+1}}\left[U\left[Y_{i+1}\right]\right] \subseteq l_{s_{i}}\left[U\left[Y_{i}\right]\right]=\operatorname{Im}\left(l_{i}^{\prime}\right)$. Pour tout $x$ de $Y_{i}$, on a $V\left(f_{m_{0}} \ldots \frown m_{i}, 0 \frown n_{0} \ldots \frown n_{i}(x)\right)=l_{s_{i}}(U(x))$. Par suite, si $y=U(x)$ et $y^{\prime}=U\left(x^{\prime}\right)$ avec $x, x^{\prime} \in Y_{i}, l_{i}^{\prime}(y)=l_{i}^{\prime}\left(y^{\prime}\right)$ entraîne successivement que

$$
V\left(f_{m_{\widehat{0}} \ldots \frown m_{i}, 0 \frown n_{\widehat{0}} \ldots \frown n_{i}}(x)\right)=V\left(f_{m_{0}} \ldots \frown m_{i}, 0 \frown n_{\widehat{0}} \ldots \frown n_{i}\left(x^{\prime}\right)\right),
$$

que

$$
f_{m_{0} \ldots \frown m_{i}, 0 \frown n_{0} \ldots \frown n_{i}}(x)=f_{m_{0}} \ldots \frown m_{i}, 0 \frown n_{\widehat{0}} \ldots \frown n_{i}\left(x^{\prime}\right),
$$

que $x=x^{\prime}$ et $y=y^{\prime}$. D'où l'injectivité de $l_{i}^{\prime}$. Le lemme 14 peut donc s'appliquer et donne la contradiction cherchée.

\section{Références}

[HKL] L. A. Harrington, A. S. Kechris and A. Louveau, A Glimm-Effros dichotomy for Borel equivalence relations, J. Amer. Math. Soc. 3 (1990), 903-928.

[Ke] A. S. Kechris, Classical Descriptive Set Theory, Springer-Verlag, 1995.

[Ku] K. Kuratowski, Topology, Vol. 1, Academic Press, New York and London, 1966.

[Le1] D. Lecomte, Classes de Wadge potentielles et théorèmes d'uniformisation partielle, Fund. Math. 143 (1993), 231-258.

[Le2] -, Uniformisations partielles et critères à la Hurewicz dans le plan, Trans. Amer. Math. Soc. 347 (1995), 4433-4460.

[Le3] -, Tests à la Hurewicz dans le plan, Fund. Math. 156 (1998), 131-165.

[Lo1] A. Louveau, Ensembles analytiques et boréliens dans les espaces produit, Astérisque 78 (1980).

[Lo2] -, A separation theorem for $\Sigma_{1}^{1}$ sets, Trans. Amer. Math. Soc. 260 (1980), $363-378$.

[Lo3] - livre à paraître.

[Lo-SR] A. Louveau and J. Saint Raymond, Borel classes and closed games: Wadgetype and Hurewicz-type results, Trans. Amer. Math. Soc. 304 (1987), 431-467.

[Mo] Y. N. Moschovakis, Descriptive Set Theory, North-Holland, 1980.

[SR] J. Saint Ray mond, La structure borélienne d'Effros est-elle standard?, Fund. Math. 100 (1978), 201-210.

Equipe d'Analyse

Université Paris 6

Tour 46-0, Boîte 186

4 Place Jussieu

75252 Paris Cedex 05, France

E-mail: lecomte@moka.ccr.jussieu.fr 